ARTICLE

DOI: $10.1038 / s 41467-017-00675-5$

\title{
Oxytocin stimulates hippocampal neurogenesis via oxytocin receptor expressed in CA3 pyramidal neurons
}

\author{
Yu-Ting Lin ${ }^{1,2}$, Chien-Chung Chen ${ }^{1}$, Chiung-Chun Huang ${ }^{1}$, Katsuhiko Nishimori ${ }^{3}$ \& Kuei-Sen Hsu (i) ${ }^{1,2,4}$
}

In addition to the regulation of social and emotional behaviors, the hypothalamic neuropeptide oxytocin has been shown to stimulate neurogenesis in adult dentate gyrus; however, the mechanisms underlying the action of oxytocin are still unclear. Taking advantage of the conditional knockout mouse model, we show here that endogenous oxytocin signaling functions in a non-cell autonomous manner to regulate survival and maturation of newly generated dentate granule cells in adult mouse hippocampus via oxytocin receptors expressed in CA3 pyramidal neurons. Through bidirectional chemogenetic manipulations, we also uncover a significant role for CA3 pyramidal neuron activity in regulating adult neurogenesis in the dentate gyrus. Retrograde neuronal tracing combined with immunocytochemistry revealed that the oxytocin neurons in the paraventricular nucleus project directly to the CA3 region of the hippocampus. Our findings reveal a critical role for oxytocin signaling in adult neurogenesis.

\footnotetext{
${ }^{1}$ Department of Pharmacology, College of Medicine, National Cheng Kung University, Tainan 70101, Taiwan. ${ }^{2}$ Institute of Basic Medical Sciences, College of Medicine, National Cheng Kung University, Tainan 70101, Taiwan. ${ }^{3}$ Department of Molecular and Cell Biology, Graduate School of Agricultural Science, Tohoku University, Miyagi 981-8555, Japan. ${ }^{4}$ Ph.D. Program for Neural Regenerative Medicine, College of Medical Science and Technology, Taipei Medical University, Taipei 11031, Taiwan. Correspondence and requests for materials should be addressed to K.-S.H. (email: richard@mail.ncku.edu.tw)
} 
O xytocin (OXT) is a nine amino acid neuropeptide that is primarily synthesized in magnocellular neurons of the hypothalamic paraventricular (PVN) and supraoptic nuclei $(\mathrm{SON})^{1,2}$. Apart from its release into the systemic circulation via the posterior pituitary, OXT is also transported axonally from hypothalamic parvocellular neurons to numerous extrahypothalamic OXT receptor (OXTR)-expressing brain regions, including the hippocampus, amygdala, lateral septum, striatum, and bed nucleus of the stria terminals, acting as either a neuromodulator or neurotransmitter to regulate neurotransmission within these regions ${ }^{3-7}$. OXT has both peripheral and central functions. Peripheral OXT promotes uterine contractions during parturition and milk ejection during lactation ${ }^{8,9}$. Centrally acting OXT has been shown to regulate various social (e.g., aggression, affiliation, bonding, and social recognition) and nonsocial behaviors (e.g., anxiety, stress, depression, and learning and memory) ${ }^{6}, 10-12$. Furthermore, OXT mediates its biological activities by binding to the OXTR, which belongs to the superfamily of G-protein-coupled receptors ${ }^{2}$.

Growing evidence suggests that the hippocampus is one of the brain structures particularly vulnerable to the effects of OXT. For example, microinjection of OXT into the dorsal hippocampus has been shown to attenuate stress-induced neuroendocrine and behavioral responses in rats ${ }^{13}$. In addition, we and others have previously shown that OXT can promote the maintenance of long-term potentiation (LTP) in hippocampal CA1 region and enhance spatial memory during motherhood ${ }^{14},{ }^{15}$. The OXT-induced enhancement of LTP is associated with a rapid and persistent increase in dendritic protein kinase $M \zeta$ protein synthesis via a mammalian target of rapamycin-mediated mechanism ${ }^{15}$. Moreover, Tsien and his colleagues have recently shown that OXT can enhance hippocampal spike transmission by increasing fast-spiking GABAergic interneuron activity to improve the performance of neural circuitry that demands synaptic specificity and temporal precision ${ }^{16}$. Despite these study points toward a crucial role for OXT in facilitating hippocampal plasticity and function, exogenous application of OXT has been reported to exert a neurotrophic effect to increase adult neurogenesis even when experienced to stressful situations ${ }^{17}$. Nonetheless, how OXT regulates adult neurogenesis remains an unresolved problem. Furthermore, it is not yet clear whether endogenous OXT signaling may also play a role in regulating adult hippocampal neurogenesis. In this study, we addressed the following three questions. First, is OXTR expressed in adult hippocampal neural progenitor cells? Second, does endogenous OXT signaling regulates specific stages of adult hippocampal neurogenesis? Finally, does endogenous OXT signaling regulates adult neurogenesis through a cell autonomous or non-cell autonomous mechanism? Using a Cre/loxP recombinase-based strategy to delete Oxtr, we report here that endogenous OXT signaling controls adult hippocampal neurogenesis through an indirect non-cell autonomous mechanism by OXTR expressed in CA3 pyramidal neurons.

\section{Results}

OXTR is not expressed in the neural progenitor cells. Adult neurogenesis is a multistep process that comprises the proliferation of neural progenitor cells, newborn neuron differentiation, maturation, and functional integration into the preexisting neural networks ${ }^{18,19}$. We first examined OXTR expression in developing dentate granule cells (DGCs) in adult mouse hippocampus by staining for proteins expressed at distinct stages of cell differentiation. Because an immunofluorescent staining using antibody is not a reliable technique for identifying OXTR-expressing neurons in mice ${ }^{20}$, we therefore used OXTR-Venus knock-in
$\left(\mathrm{Oxtr} \mathrm{Venus}^{\mathrm{N}} \mathrm{Neo} /+\right)$ mice to characterize OXTR-expressing neurons in the hippocampus. In 10-week-old mice, unexpectedly, we found few or no Venus-positive cells in the dentate gyrus (DG) expressing progenitor markers, such as nestin (Fig. 1a), Ki67 (Fig. 1b), or neuroblast and immature neuronal marker doublecortin (DCX; Fig. 1c). However, in virtually all Venus-positive cells coexpressed the mature neuronal-specific nuclear protein (NeuN; Fig. 1d). Immunofluorescence staining was also performed with antibodies against numerous known marker proteins expressed in the mature granule cell (calbindin) and the subtypes of GABAergic interneurons (calretinin and parvalbumin). In the hilus, $77.4 \pm 2.3 \%$ of Venus-positive cells expressed calretinin immunoreactivity (Fig. 1e) and $25.0 \pm 2.9 \%$ Venus-positive cells expressed parvalbumin immunoreactivity (Fig. 1f). However, no Venus-positive cells were found that expressed calbindin (Fig. 1g). To label proloferating cells, we administered a synthetic analog of the nucleoside thymidine 5-bromo-2'-deoxyuridine (BrdU) intraperitoneally and analyzed BrdU incorporation 2 or 4 weeks later. We detected no Venus-positive cells expressing BrdU immunoreactivity (Fig. 1h, i). We further characterize hilar and CA3 OXTR distribution regarding to overlap with GABA immunoreactivity. In the hilus, $27.8 \pm 3.8 \%$ of Venus-positive cells were immunoreactive for the GABAergic marker glutamic acid decarboxylase (GAD)67. In the CA3, 3.8 $\pm 0.7 \%$ of Venuspositive cells expressed GAD67 immunoreactivity (Supplementary Fig. 1a, b). These results indicate that OXTR is not expressed in the neural progenitor cells that reside within the subgranular zone or mature granule cells of adult DG and a small subset of hilar GABAergic neurons express OXTR.

Deletion of Oxtr from hippocampal excitatory neurons. Previous studies have shown that CA3 pyramidal neurons send reciprocal projections back to the DG and regulate neurogenesis in the DG of adult rats ${ }^{21}$. Since OXTR is enriched in the CA2 and CA3 of the hippocampus ${ }^{20}$, we therefore hypothesized that OXT may control adult hippocampal neurogenesis via OXTR expressed in CA3 pyramidal neurons. Consistent with previous findings $^{20}$, our immunofluorescent staining data showed strong Venus immunoreactivity in the hippocampal CA3 region of Oxtr Venus-Neo/t+ mice. We found that more than 95\% Venuspositive cells expressed calcium/calmodulin-dependent protein kinase II $\alpha(\mathrm{CaMKII} \alpha)$ immunoreactivity (Fig. 2a), indicating that OXTR predominantly expressed in excitatory pyramidal neurons. To test whether OXTR expressed in CA3 pyramidal neurons plays a role in regulating adult DG neurogenesis, we used the Cre-loxP recombination approach to conditionally delete Oxtr from hippocampal excitatory neurons by crossing mice expressing CaMKII $\alpha$-Cre with mice in which Oxtr is floxed (Oxtr f/f). Polymerase chain reaction (PCR) screening of mouse genomic tail DNA confirmed heterozygous $\left(\mathrm{Oxtr}^{+{ }^{+}}\right)$and homozygous Oxtr $\left(\mathrm{Oxtr}^{-/-}\right)$conditional knockout mice (Fig. 2b). Quantitative real-time PCR analysis confirmed a reduction in Oxtr mRNA expression in the CA2, CA3, and hypothalamus, but not the hilus of the DG, in $\mathrm{Oxtr}^{-/-}$mice compared with wild-type (WT, Oxtrf/f) mice (Fig. 2c). In parallel, fluorescence in situ hybridization (FISH) with Oxtr gene probe also revealed that the numbers of Oxtr mRNA-positive cells in the CA2 and CA3 of $\mathrm{Oxtr}^{-1}$ mice were markedly reduced compared with WT mice (Fig. 2d, e), confirming the efficiency of Cre-loxP-mediated deletion of Oxtr. Dual-probe FISH also confirmed that the majority of Oxtr mRNA-positive cells were CaMKII $\alpha$ mRNAexpressing cells in the CA3 (Supplementary Fig. 2a). Very few Oxtr mRNA immunoreactivity was detected in the granule cell layer of the dorsal and ventral DG (Supplementary Fig. 2b, c). Although Oxtr mRNA immunoreactivity was detected in dorsal 
a

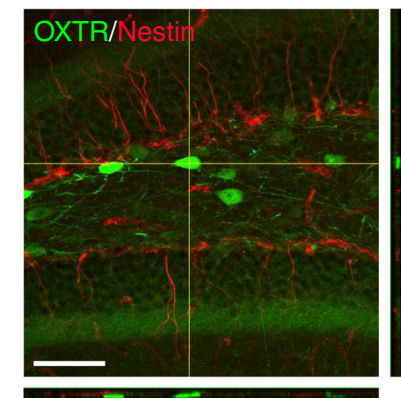

d

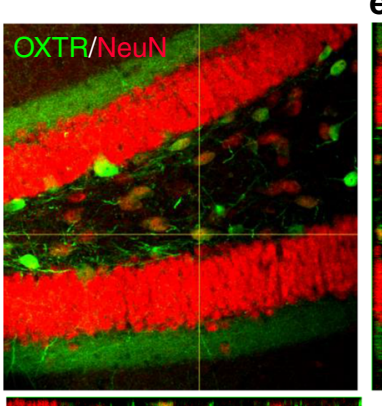

$\mathbf{g}$

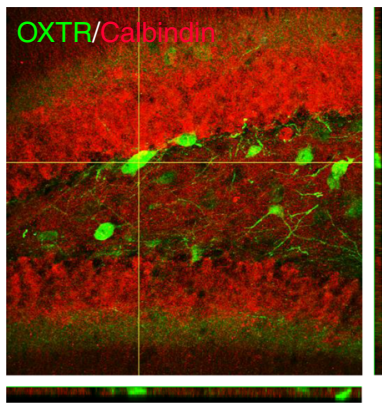

b

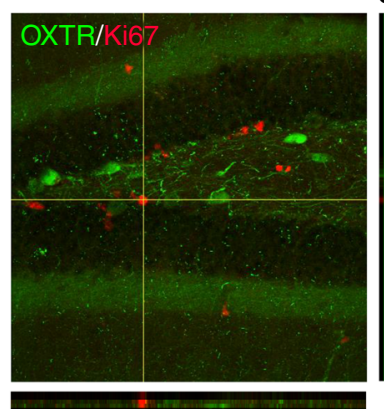

e

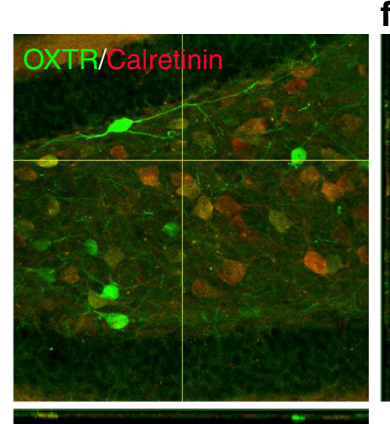

h

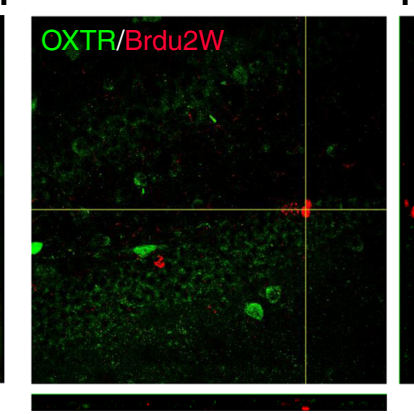

i
C

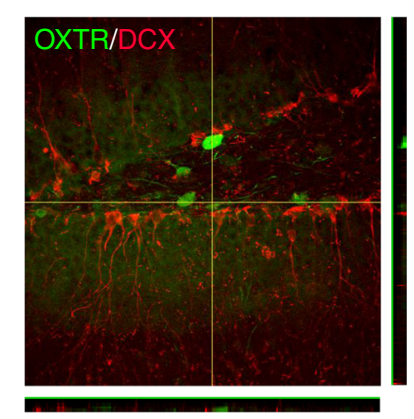

$\mathbf{f}$
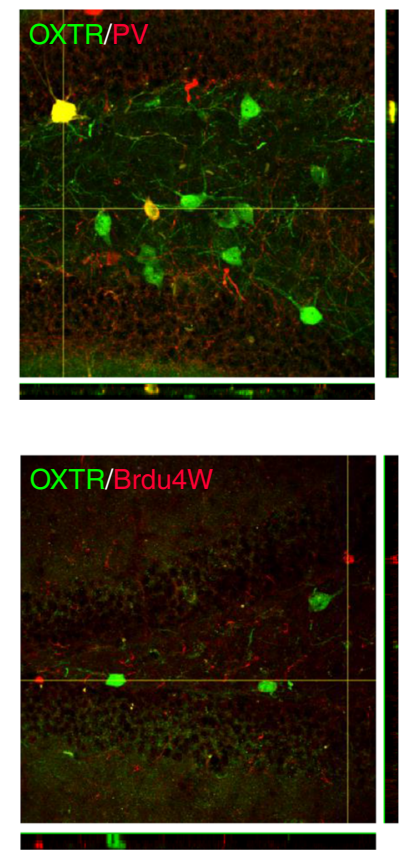

Fig. 1 Expression of OXTR in the DG. a-g Double-labeled confocal immunofluorescence images showing the colocalization of OXTR (green) expression with a nestin (red), b Ki67 (red), c DCX (red), d NeuN (red), e calretinin (red), f parvalbumin (PV, red), and $\mathbf{g}$ calbindin (red) in the DG of 10-week-old OxtrVenus$\mathrm{Neo/+}$ mice. h, i Immunofluorescent staining of BrdU. Oxtr ${ }^{\mathrm{Venus}-\mathrm{Neo} /+}$ mice were injected with BrdU and killed 2 or 4 weeks later. Representative images of $\mathrm{BrdU}^{+}$cells at $\mathbf{h} 2$ week and $\mathbf{i} 4$ weeks. Data was replicated in four mice. Scale bar, $50 \mu \mathrm{m}$

and ventral hilus of the DG, we did not observe colocalization of Oxtr mRNA with CaMKII $\alpha$ mRNA. We also demonstrated that $\mathrm{Oxtr}^{-1-}$ mice specifically displayed a reduction of Oxtr mRNA expression in the CA3, but not the hilus of the DG, compared with WT mice (Supplementary Fig. 2).

Effects of Oxtr deletion on newly generated DGCs. To determine whether Oxtr deletion from hippocampal excitatory neurons may affect the proliferation of newly generated DGCs, WT and $\mathrm{Oxtr}^{-/-}$mice were given a single injection of BrdU, and double fluorescent labeling for detection of BrdU-positive (BrdU ${ }^{+}$) proliferating cells $\left(\mathrm{Ki}_{67}{ }^{+}\right)$was performed on both dorsal and ventral hippocampal sections $2 \mathrm{~h}$ later (Fig. 3a, c). Stereological analysis revealed no differences in the total number of either $\mathrm{BrdU}^{+}$(Fig. 3d) or Ki67 ${ }^{+}$cells (Fig. 3e) between WT and $\mathrm{Oxtr}^{-/}$ mice at $2 \mathrm{~h}$ after the BrdU injection in both dorsal and ventral hippocampus (Supplementary Fig. 3a-d). The percentage of $\mathrm{BrdU}^{+} \mathrm{Ki} 7^{+}$cells to total $\mathrm{BrdU}^{+}$cells was not different between the two groups (Supplementary Fig. 3i). To determine whether conditional deletion of Oxtr from hippocampal excitatory neurons may affect the survival of newly generated DGCs, WT and $\mathrm{Oxtr}^{-1-}$ mice were subjected to multiple BrdU injections, and double fluorescent labeling for detection of $\mathrm{BrdU}^{+}$immature $\left(\mathrm{DCX}^{+}\right)$or mature neuronal cells $\left(\mathrm{NeuN}^{+}\right)$was performed on hippocampal sections 14 or 28 days later (Fig. 3b, c). Interestingly, we found a significant reduction in the total number of $\mathrm{BrdU}^{+}$cells in $\mathrm{Oxtr}^{-1-}$ mice at 14 and 28 days after BrdU injection compared with WT mice (Fig. 3f). Likewise, a significant reduction in the total number of $\mathrm{DCX}^{+}$cells in $\mathrm{Oxtr}^{-1-}$ mice at 14 days after BrdU injection compared with WT mice in both dorsal and ventral hippocampus (Fig. $3 \mathrm{~g}$ and Supplementary Fig. 3e-h). However, the percentage of $\mathrm{BrdU}^{+} \mathrm{DCX}^{+}$cells or $\mathrm{BrdU}^{+} \mathrm{NeuN}^{+}$cells to total $\mathrm{BrdU}^{+}$cells was not different between two groups (Supplementary Fig. 3j, k). These results suggest that OXTR plays a non-cell autonomous role in controlling the survival of newly generated DGCs, whereas the progenitor cell proliferation and early differentiation into immature neurons were unaltered by Oxtr deletion.

We next evaluated the impact of Oxtr deletion on the morphological maturation of newly generated DGCs by using a retrovirus-mediated birth-dating and labeling strategy ${ }^{22}$. Engineered retroviruses expressing enhanced green fluorescent protein (EGFP) were stereotaxically microinjected into the DG hilus of WT and $\mathrm{Oxtr}^{-/}$mice. Sholl analysis for dendritic complexity of EGFP-positive $\left(\mathrm{EGFP}^{+}\right)$DGCs was carried out 14 
a
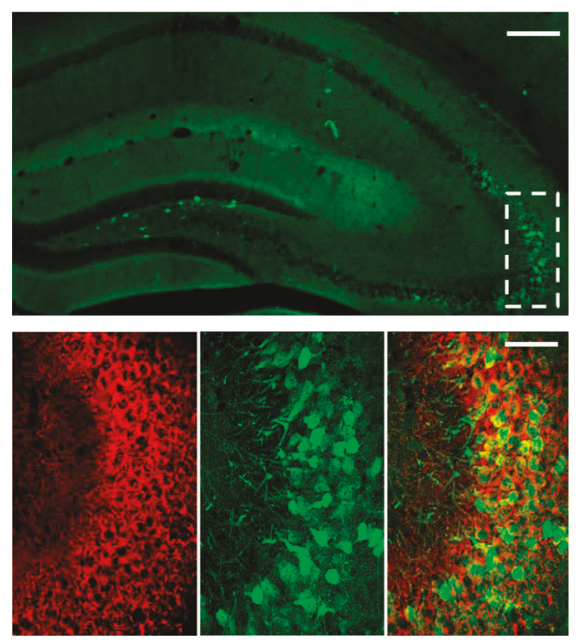

CaMKIlo

d

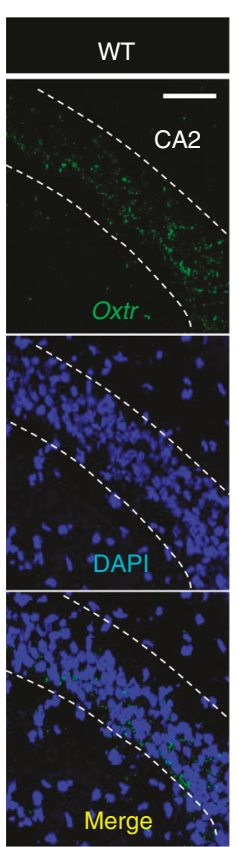

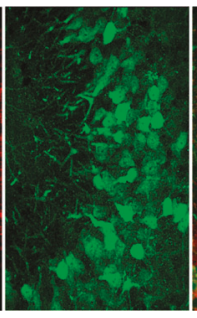

OXTR-Venus

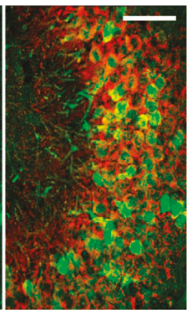

Merge b
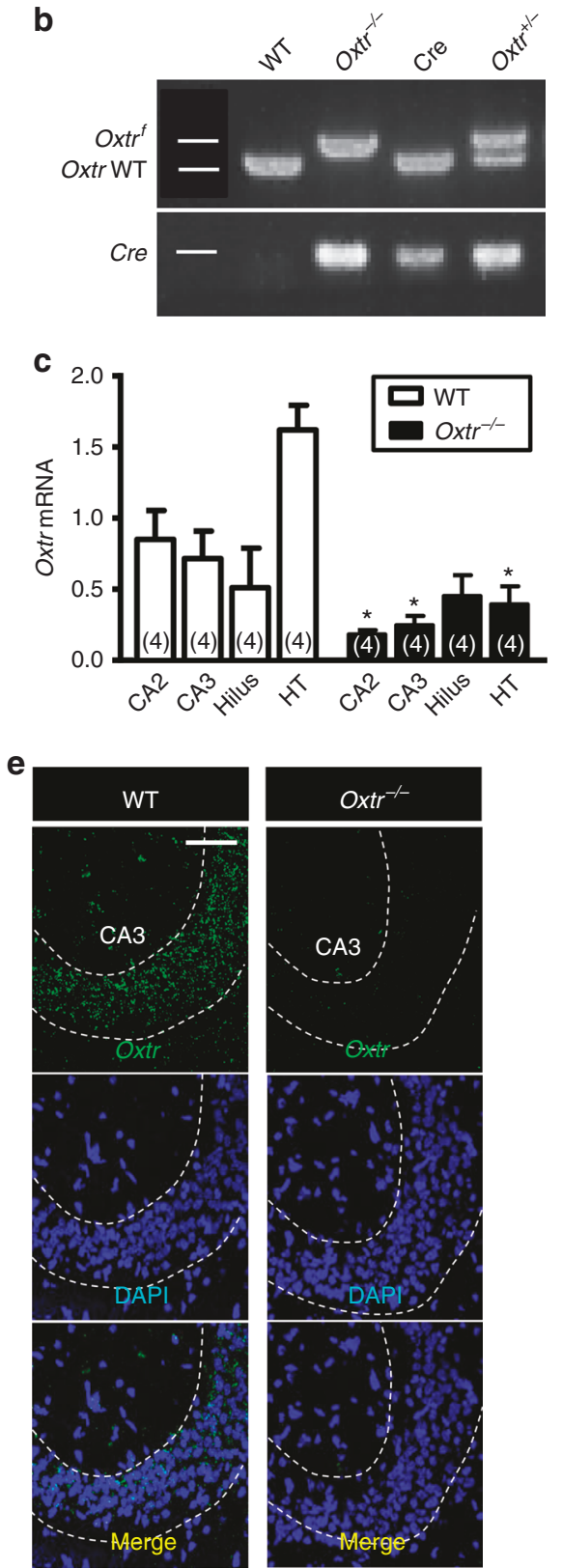

Fig. 2 Conditional deletion of Oxtr in the excitatory neurons of mouse hippocampus. a Top panel, a representative immunofluorescence image of OXTR (green) expression in the mouse hippocampus. Bottom panels, augmented figures showing CaMKIl $\alpha$ (red) and OXTR (green) double-labeling results in rectangle area. Scale bars: top, $200 \mu \mathrm{m}$; bottom, $50 \mu \mathrm{m}$. b PCR screening of tail-derived genomic DNA for selection of Oxtr ${ }^{-/-}$mice. c Quantitative real-time PCR of Oxtr mRNA in the CA2, CA3, hilus, and hypothalamus $(H T)$ regions from WT (Oxtr $\left.f^{f / f}\right)$ and Oxtr ${ }^{-/-}$mice $\left(n=4\right.$ mice for each group; ${ }^{\star} P<0.05$, unpaired two-tailed Student's $t$ test). d, e FISH images showing the expression of Oxtr mRNA in the CA2 d and CA3 regions e of WT and Oxtr ${ }^{-/-}$mice (counterstained with DAPI, blue). Scale bar, $50 \mu \mathrm{m}$. Data was replicated in four mice

or 28 days post infection (dpi). Using confocal microscopy to reconstruct the dendritic arborization of $\mathrm{EGFP}^{+}$DGCs, we found that newly generated DGCs from $\mathrm{Oxtr}^{-1-}$ mice exhibited less elaborated dendritic arborization than those from WT mice at 14 dpi (Fig. 4a). Unpaired Student's $t$ test revealed a significant reduction in both total dendritic length $(P<0.001$; Fig. $4 \mathrm{~b})$ and branch number $(P<0.01$; Fig. $4 \mathrm{c})$ in $\mathrm{EGFP}^{+}$DGCs from $\mathrm{Oxtr}^{-/-}$ mice compared to those from WT mice. Sholl analysis further revealed a significant decrease in the dendritic complexity of EGFP $^{+}$DGCs in $\mathrm{Oxtr}^{-/-}$mice compared to WT mice at $14 \mathrm{dpi}$, an effect more pronounced for the dendritic branches extended beyond $90-110 \mu \mathrm{m}$ from the soma (Fig. 4d). However, we observed no significant differences in total dendritic length, branch number, or dendritic complexity of $\mathrm{EGFP}^{+}$DGCs between $\mathrm{Oxtr}^{-/}$and WT mice at $28 \mathrm{dpi}$ (Fig. $4 \mathrm{e}-\mathrm{h}$ ).

To further confirm OXT controls adult hippocampal neurogenesis through an indirect non-cell autonomous mechanism, we therefore examined morphological maturation of newly generated DGCs by using Oxtrf/f mice in combination with Cre recombinase-mediated gene deletion in a localized fashion through bilateral stereotaxic injections of Cre-expressing (RetroCre-EGFP) or control EGFP-expressing (Retro-EGFP) retroviral vectors targeting the hilus of the DG (Fig. 4i). We observed no significant differences in total dendritic length or dendritic complexity of $\mathrm{EGFP}^{+}$DGCs between two groups at $14 \mathrm{dpi}$ (Fig. 4j, k). 
To assess whether the morphological changes that resulted from conditional Oxtr deletion may lead to alterations in physiological properties, we performed electrophysiological analysis of newly generated DGCs in acute slices from retrovirus-injected mice at $14 \mathrm{dpi}$. We first examined glutamatergic synaptic transmission by recording spontaneous excitatory postsynaptic currents (sEPSCs) in $\mathrm{EGFP}^{+}$DGCs in the presence of $\mathrm{GABA}_{\mathrm{A}}$ receptor antagonist gabazine $(10 \mu \mathrm{M}$; Fig. 5a). A significant difference in cumulative interevent interval distribution was observed between newly generated DGCs from $\mathrm{Oxtr}^{-/-}$ and WT mice (Fig. 5b). However, no significant difference in cumulative amplitude distribution was observed between two groups (Fig. 5c). The mean frequency of sEPSCs in EGFP ${ }^{+}$DGCs from $\mathrm{Oxtr}^{-/}$mice was significantly less than those from WT mice $(P<0.05$; Fig. $5 \mathrm{~d})$. No significant difference was observed between $\mathrm{Oxtr}^{-/-}$and WT mice in the mean amplitude of sEPSCs $(P=0.88$; Fig. 5e).

The newly generated DGCs exhibit initial depolarizing response to GABA, which gradually shift to hyperpolarizing responses over a period of 2-3 weeks of birth ${ }^{23}$. The polarity of GABA actions depends on the chloride concentration gradient across the plasma membrane. We next used gramicidin-perforated patch-clamp recordings to determine the nature of GABA activation by recording evoked inhibitory postsynaptic currents (IPSCs) in $\mathrm{EGFP}^{+}$DGCs from Oxtr ${ }^{-1-}$ and WT mice at 10,14 , and $28 \mathrm{dpi}$ (Fig. 5f). In agreement with previous findings ${ }^{23}$, we found that the reversal potential for $\mathrm{GABA}_{\mathrm{A}}$ receptor-mediated IPSCs $\left(\mathrm{E}_{\mathrm{GABA}}\right)$ in $\mathrm{EGFP}^{+}$DGCs gradually decreased during maturation. Two-way ANOVA revealed a main effect of conditional Oxtr deletion on $\mathrm{E}_{\mathrm{GABA}}\left(F_{(1,18)}=25.1, P<0.001\right.$; Fig. $\left.5 \mathrm{~g}\right)$. Bonferroni's post hoc tests showed that EGFP ${ }^{+}$DGCs from Oxtr ${ }^{-/-}$mice demonstrated more positive values for $\mathrm{E}_{\mathrm{GABA}}$ compared to those from WT mice at both $10(P<0.01)$ and $14 \mathrm{dpi}(P<0.05)$. To determine whether more positive values for $\mathrm{E}_{\mathrm{GABA}}$ could be due to higher intracellular chloride concentrations $\left(\left[\mathrm{Cl}^{-}\right]_{\mathrm{i}}\right)$, we used the Nernst equation to calculate $\left[\mathrm{Cl}^{-}\right]_{\mathrm{i}}$. Two-way ANOVA revealed a main effect of conditional Oxtr deletion on $\left[\mathrm{Cl}^{-}\right]_{\mathrm{i}}\left(F_{(1,18)}=23.7, P<0.01\right.$; Fig. 5h). Bonferroni's post hoc tests showed that $\mathrm{GFP}^{+}$DGCs from $\mathrm{Oxtr}^{-/-}$ mice had higher $\left[\mathrm{Cl}^{-}\right]_{\mathrm{i}}$ than those from WT mice at both 10 $(P<0.0001)$ and $14 \mathrm{dpi}(P<0.01)$. These results indicate that conditional Oxtr deletion may delay maturation of newly generated DGCs.

We next evaluated whether Oxtr deletion impacted mature DGCs. To characterize the morphological and
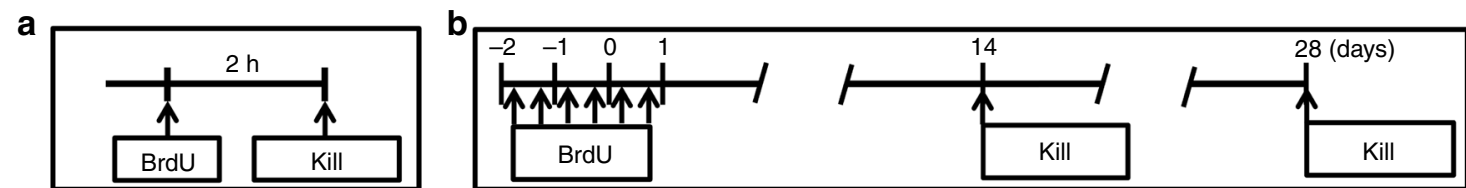

C
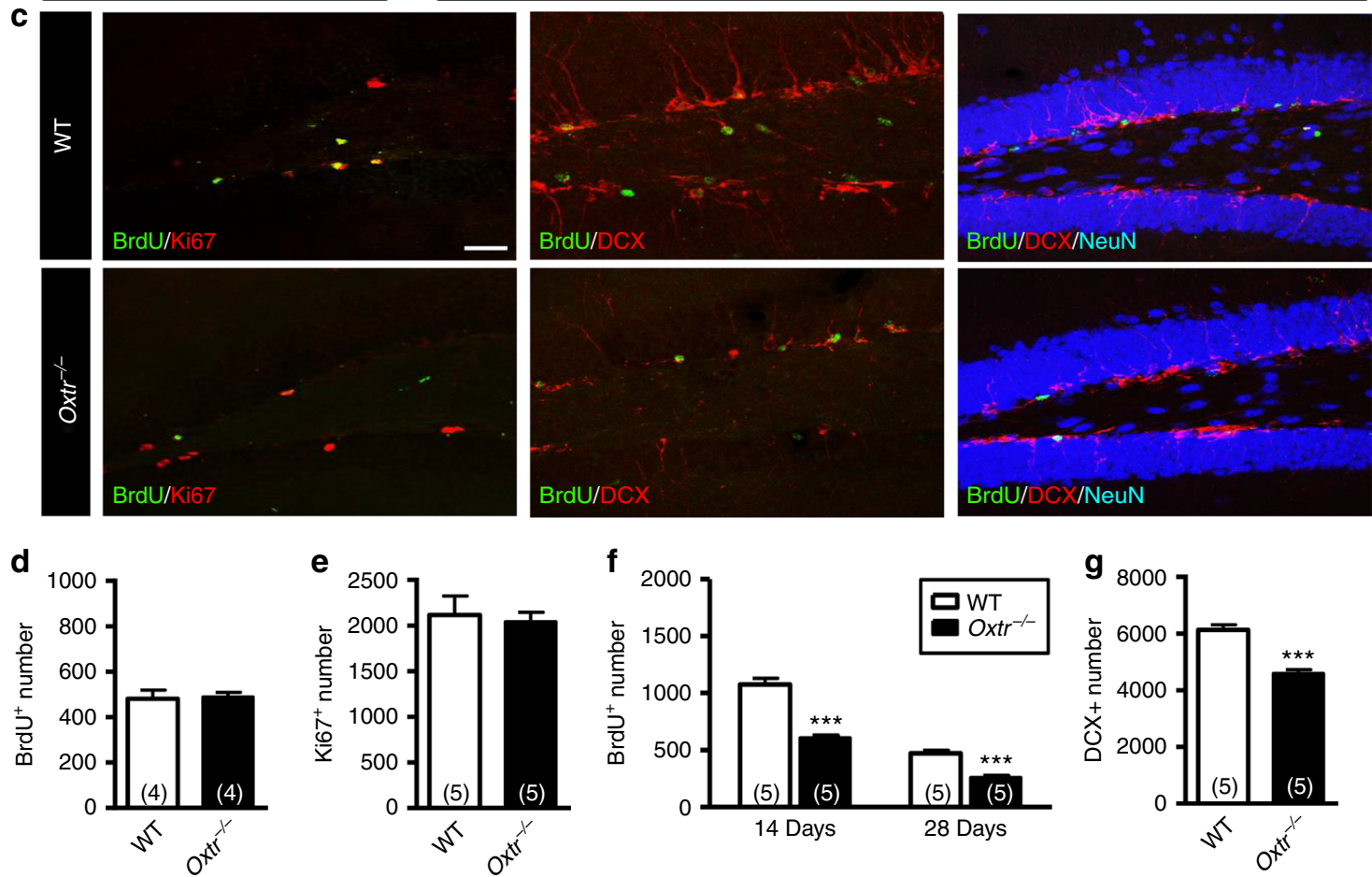

Fig. 3 Conditional deletion of Oxtr impairs the survival of newly generated DGCs. a Schematic representation of the experimental designs for comparing the proliferation of newly generated DGCs in WT and Oxtr ${ }^{-/-}$mice. Mice were given a single injection of BrdU $(50 \mathrm{mg} / \mathrm{kg})$ and were killed $2 \mathrm{~h}$ after BrdU injection. $\mathbf{b}$ Schematic representation of the experimental designs for comparing the survival of newly generated DGCs in WT and Oxtr ${ }^{-/-}$mice. Mice were injected six times intraperitoneally with BrdU $(50 \mathrm{mg} / \mathrm{kg})$ at $12 \mathrm{~h}$ intervals and were killed 14 and 28 days after the last BrdU injection. c Representative immunofluorescence images of hippocampal sections from WT and Oxtr ${ }^{-/-}$mice double or triple stained for BrdU (green), Ki67 (red), DCX (red), and NeuN (blue) after BrdU injection. Scale bar, $100 \mu \mathrm{m}$. d Quantification of the total number of BrdU ${ }^{+}$cells in the DG of WT and Oxtr ${ }^{-/-}$mice at $2 \mathrm{~h}$ after BrdU injection ( $n=4$ mice per genotype, unpaired two-tailed Student's $t$ test). e Quantification of the total number of Ki67 ${ }^{+}$cells in the DG of WT and Oxtr ${ }^{-/-}$ mice at $2 \mathrm{~h}$ after BrdU injection ( $n=5$ mice per genotype, unpaired two-tailed Student's $t$ test). $\mathbf{f}$ Quantification of the total number of BrdU ${ }^{+}$cells in the DG of WT and $O x t^{-1-}$ mice at 14 and 28 days after BrdU injection ( $n=5$ mice for each group; ${ }^{\star \star \star} P<0.001$, unpaired two-tailed Student's $t$ test).

g Quantification of the total number of DCX $X^{+}$cells in the DG of WT and Oxtr ${ }^{-/-}$mice at 14 days after the last BrdU injection ( $n=5$ mice per genotype; ${ }^{\star \star \star} P<0.001$, unpaired two-tailed Student's $t$ test). Data represent the mean \pm s.e.m 
electrophysiological properties of DGCs, we recorded miniature excitatory postsynaptic currents (mEPSCs) from mature DGCs with the use of whole-cell patch-clamp recordings and biocytin was routinely included in the intracellular solution to allow post hoc identification of the recorded neurons (Fig. 6a). We observed no significant differences between $\mathrm{Oxtr}^{-/-}$and WT mice in total

a
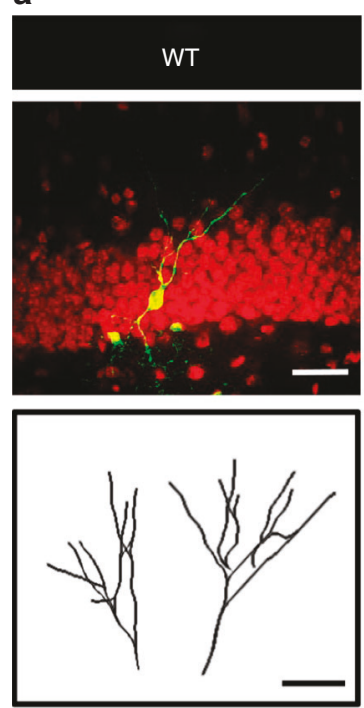

e

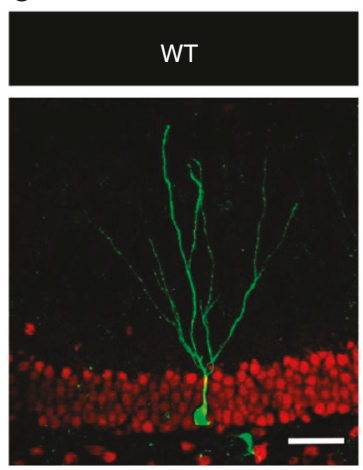

i
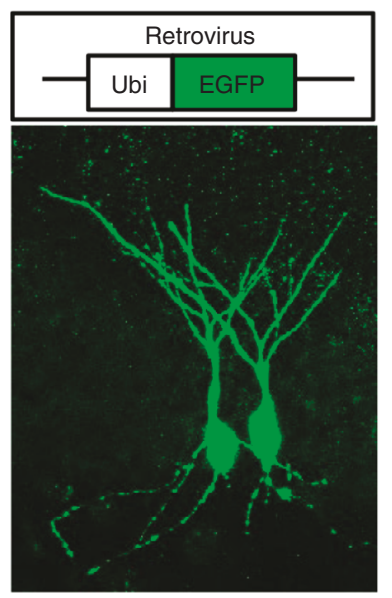
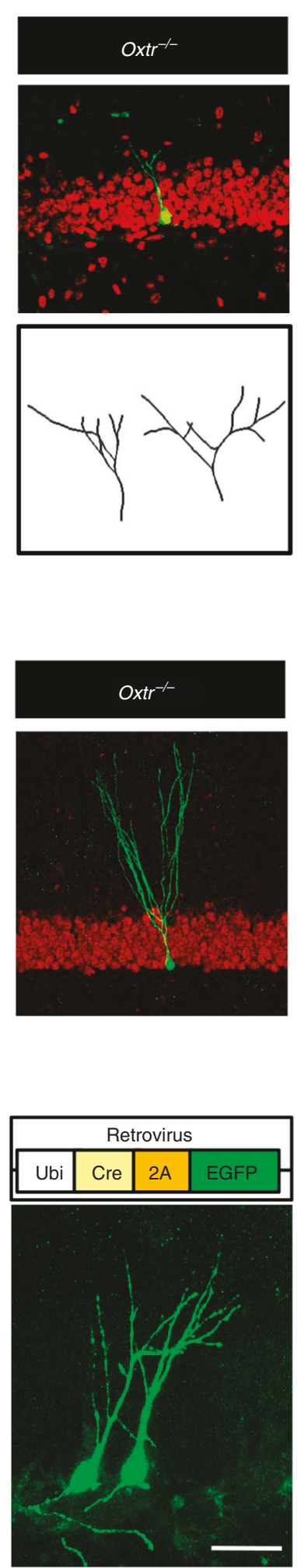

dendritic length $(P=0.19$; Fig. $6 \mathrm{~b})$ and branch number of DGCs $(P=0.22$; Fig. $6 \mathrm{c})$. Sholl analysis revealed no significant difference in the dendritic complexity of DGCs between $\mathrm{Oxtr}^{-1-}$ and WT mice $\left(F_{(1,225)}=0.14, P=0.71\right.$; Fig. $\left.6 \mathrm{~d}\right)$. mEPSCs were recorded in the presence of tetrodotoxin (TTX, $0.5 \mu \mathrm{M})$ and gabazine $(10 \mu \mathrm{M}$; Fig. 6e). A significant difference in cumulative interevent interval


f

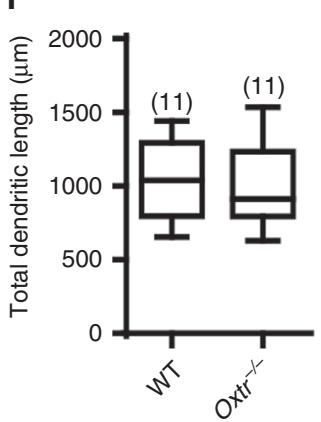

g
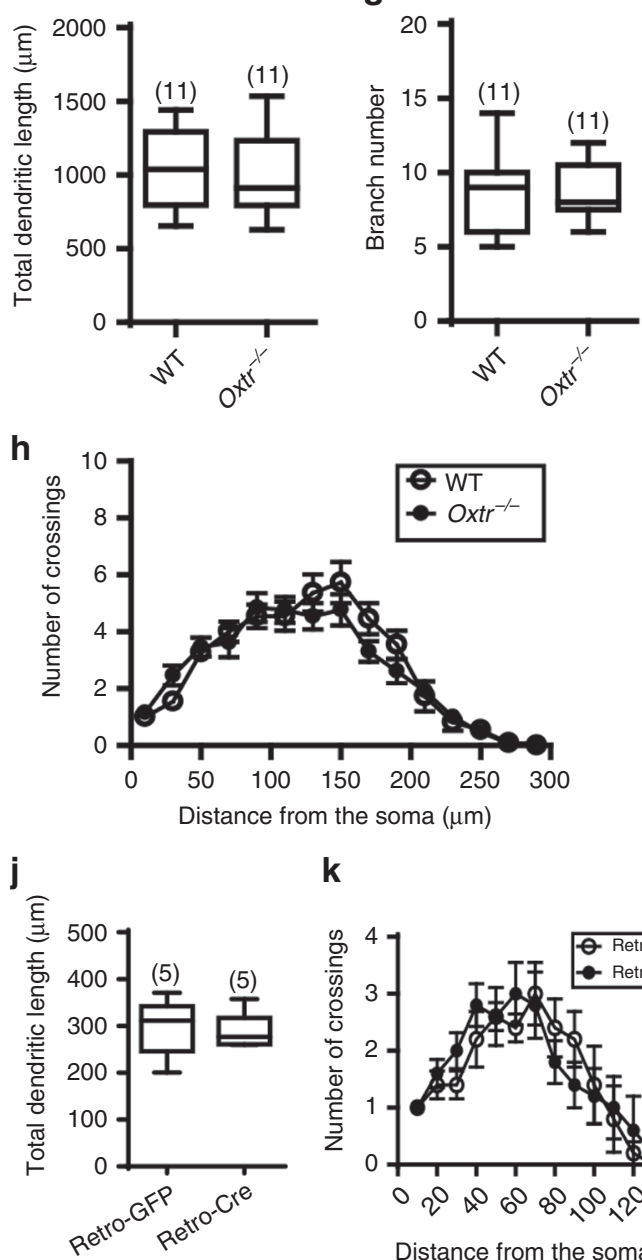

k

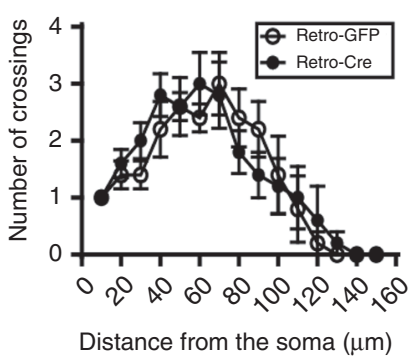


distribution was observed between mature DGCs from $\mathrm{Oxtr}^{-/}$ and WT mice (Fig. 6f). However, no significant difference in cumulative amplitude distribution was observed between two groups (Fig. 6g). The mean frequency of mEPSCs in DGCs from $\mathrm{Oxtr}^{-/-}$mice was significantly less than those from WT mice $(P<0.05$; Fig. 6h). No significant difference was observed between $\mathrm{Oxtr}^{-/-}$and WT mice in the mean amplitude of mEPSCs $(P=0.11$; Fig. 6i).

OXT controls adult neurogenesis through CA3 neurons. Having established that conditional deletion of Oxtr results in impaired survival and maturation of newly generated DGCs, we next investigated the possible mechanisms by which OXT controls adult hippocampal neurogenesis. Based on the foregoing observations in conjunction with the suggested role of CA3 pyramidal neurons in regulating adult neurogenesis, we tested whether OXT neurons of the PVN send projections to the CA3 region of the hippocampus. To identify PVN-CA3 projection neurons, we injected a monosynaptic retrograde tracer cholera toxin $\mathrm{B}$ subunit (CTB) into the $\mathrm{CA} 3$ and examined the distribution of retrogradely CTB-labeled cells appeared in the PVN (Fig. 7a, b). We also labeled neurosecretory neurons with intraperitoneal injection of the retrograde tracer, fluoro-gold $(4 \% \mathrm{w} / \mathrm{v}$ in $100 \mu \mathrm{l}$ saline ${ }^{24}$. Immunoreactivity for CTB was clearly detected in fluoro-gold ${ }^{-}$and $\mathrm{OXT}^{+}$parvocellular neurons of the PVN (Fig. 7b), but not the SON (Supplementary Fig. 4a). We also observed that PVN neurons send their projecting fibers directly to the CA3 (Supplementary Fig. 4b). These results indicate that the CA3 contains a dense population of OXTR-expressing neurons and receives direct inputs from OXT neurons in the PVN.

To demonstrate that CA3 pyramidal neurons are functionally modulated by OXT, we analyzed the effects of OXT on electrical membrane properties of CA3 neurons (Fig. 7c). Under whole-cell current-clamp condition, bath application of OXT $(1 \mu \mathrm{M})$ consistently caused a membrane depolarization by $3.98 \pm 1.44$ $\mathrm{mV}(n=8)$ in CA3 pyramidal neurons from WT mice. The OXT-induced membrane depolarization was not observed in CA3 pyramidal neurons from $\mathrm{Oxtr}^{-1-}$ mice $(0.98 \pm 0.21 \mathrm{mV}, n=10$; Fig. 7d, e) and was prevented by the selective OXTR antagonist L-371257 ( $1 \mu \mathrm{M}$, Supplementary Fig. 5a, b), indicating that such action is mediated by the OXTR. Furthermore, consistent with increased CA3 pyramidal neuron excitability, we found that the firing activity of $\mathrm{CA} 3$ neurons by quantifying the number of action potentials in response to postsynaptic depolarizing current injection (100 pA, $500 \mathrm{~ms})$ was increased after OXT application (4.0 \pm 0.9 spikes, $n=10, P<0.05)$ compared with vehicle-treated controls $(1.3 \pm 0.8$ spikes, $n=10)$ in WT mice (Fig. 7f, g). However, bath application of OXT failed to elicit any significant change in the number of action potentials in response to postsynaptic depolarizing current injection in CA3 pyramidal neurons from $\mathrm{Oxtr}^{-/-}$mice $(1.6 \pm 1.3$ spikes, $n=10, P=0.22)$ compared with vehicle-treated controls (1.2 \pm 0.8 spikes, $n=10)$. The enhancing effect of OXT on action potential firing in CA3 pyramidal neurons was also prevented by the pretreatment of the hippocampal slices with L-371257 (1 $\mu$ M; Supplementary Fig. 5c, d). To further validate whether OXT may act indirectly to enhance intrinsic excitability of hippocampal interneurons through activation of CA3 OXTR $^{+}$pyramidal neurons, CA3 OXTR $^{-}$ interneurons in slices from Oxtr $\mathrm{Venus- \textrm {Neo } / +}$ mice were targeted for recording, filled with biocytin to allow post hoc reconstruction (Supplementary Fig. 6a). In contrast to what has been observed in $\mathrm{CA}^{3} \mathrm{OXTR}^{+}$pyramidal neurons, bath application of OXT $(1 \mu \mathrm{M})$ did not significantly affect membrane potential in the absence of presence of L-371257 $(1 \mu \mathrm{M})$ in CA3 OXTR $^{-}$interneurons (Supplementary Fig. 6b-d).

We employed optogenetic approach to further validate that DGCs receive a direct back projection from CA3 pyramidal neurons. We unilaterally injected the CA3 region with a recombinant adeno-associated virus capsid DJ (AAV-DJ) serotype expressing the light-sensitive channelrhodopsin 2 (ChR2) tagged with enhanced yellow fluorescent protein (EYFP), under control of the CaMKII $\alpha$ promoter [AAV-CaMKII $\alpha-h C h R 2$ (H134R)-EYFP], favoring expression within excitatory neurons ${ }^{25}$. Acute brain slices were prepared 3 weeks after virus injection. Our immunohistochemical analysis revealed robust and unilateral expression of EYFP in the subgranular zone of the DG and the stratum radiatum (Fig. 7h). We used whole-cell recording to examine postsynaptic currents in DGCs while optically stimulating ChR2-EYFP-positive projections in the DG. Blue light pulses reliably induced postsynaptic currents in DGCs. These optically evoked postsynaptic currents were not significantly affected by gabazine $(10 \mu \mathrm{M})$ but were blocked by concomitant application of 6-cyano-7-nitroquinoxaline-2, 3-dione (CNQX; 20 $\mu \mathrm{M})$ and D-2-amino-5-phosphonopentanoic acid (APV; $50 \mu \mathrm{M}$ ), indicating that they were excitatory postsynaptic responses (Fig. 7i). We confirmed that DGCs indeed receive a direct back projection from CA3 pyramidal neurons.

Given that chemogenetics permits bidirectional manipulation of neuronal activity with anatomical, genetic, and temporal precision, we also used in vivo chemogenetic approaches to study the role of CA3 pyramidal neuronal activity in controlling adult hippocampal neurogenesis. The experimental procedure is depicted in Fig. 8a. We bilaterally injected the CA3 region with an AAV-DJ viral vector expressing an engineered Gq-coupled human M3 (hM3Dq) tagged with mCitrine (hM3Dq-mCitrine) or $\mathrm{G}_{\mathrm{i} / \mathrm{o}}$-coupled human $\mathrm{M} 4$ (hM4Di) tagged with mCherry (hM4Di-mCherry), under control of the CaMKII $\alpha$ promoter. One week later, WT and $\mathrm{Oxtr}^{-/}$mice were subjected to multiple BrdU injections and analyzed BrdU incorporation 2 weeks later.

Fig. 4 Conditional deletion of Oxtr delays the morphological maturation of newly generated DGCs. a Confocal three-dimensional reconstruction of dendrites of EGFP ${ }^{+}$DGCs from WT and Oxtr ${ }^{-/-}$mice at 14 dpi. Scale bar, $50 \mu \mathrm{m}$. b, c Summary bar graphs depicting $\mathbf{b}$ the total dendritic length and $\mathbf{c}$ branch number of EGFP ${ }^{+}$DGCs from WT and Oxtr ${ }^{-/-}$mice at $14 \mathrm{dpi}\left(n=11\right.$ neurons from 4 mice per genotype; ${ }^{\star \star} P<0.01,{ }^{\star \star \star} P<0.001$, unpaired two-tailed Student's $t$ test). d Sholl analysis of dendritic complexity of EGFP ${ }^{+}$DGCs from WT and Oxtr ${ }^{-1-}$ mice at 14 dpi ( $n=11$ neurons from 4 mice per genotype; ${ }^{\star} p<0.05,{ }^{\star \star} p<0.01$, two-way ANOVA with Bonferroni's post hoc test). e Representative confocal images showing EGFP ${ }^{+}$DGCs from WT and $\mathrm{Oxtr}^{-1-}$ mice at $28 \mathrm{dpi}$. Scale bar, $50 \mu \mathrm{m}$. f, $\mathbf{g}$ Summary bar graphs depicting $\mathbf{f}$ the total dendritic length and $\mathbf{g}$ branch number of EGFP DGCs from WT and $\mathrm{Oxtr}^{-/-}$mice at $28 \mathrm{dpi}\left(n=11\right.$ neurons from 5 mice per genotype; unpaired two-tailed Student's $t$ test). $\mathbf{h}$ Sholl analysis of dendritic complexity of EGFP ${ }^{+}$ DGCs from WT and Oxtr ${ }^{-/-}$mice at $28 \mathrm{dpi}(n=11$ neurons from 5 mice per genotype; two-way ANOVA). i Top panel, schematic representations of the retroviral vectors used for in vivo genetic manipulation under the control of the Ubi-1 promoter. Bottom panels, representative confocal images showing Retro-EGFP ${ }^{+}$and Retro-Cre-2A-EGFP ${ }^{+}$DGCs in Oxtr//f mice at $14 \mathrm{dpi}$. Scale bar, $50 \mu \mathrm{m}$. $\mathbf{j}$ Summary bar graphs depicting the total dendritic length of Retro-EGFP ${ }^{+}$and Retro-Cre-2A-EGFP DGCs in Oxtr $f^{f / f}$ mice at $14 \mathrm{dpi}(n=5$ neurons from 3 mice for each group, unpaired two-tailed Student's $t$ test). k Sholl analysis of dendritic complexity of Retro-EGFP ${ }^{+}$and Retro-Cre-2A-EGFP DGCs in Oxtr f/f mice at 14 dpi $(n=5$ neurons from 3 mice for each group, two-way ANOVA). Data represent the mean \pm s.e.m 
Mice were injected intraperitoneally daily with clozapine- $N$-oxide (CNO, $10 \mathrm{mg} / \mathrm{kg}$ ), a synthetic ligand of hM3Dq and hM4Di ${ }^{26}$, for continuous 2 weeks until killig. Post hoc histological examination of brain sections revealed robust and bilateral expression of hM3Dq or hM4Di in CA3 pyramidal neurons (Fig. 8b). In a subset of mice, we performed ex vivo electrophysiological a

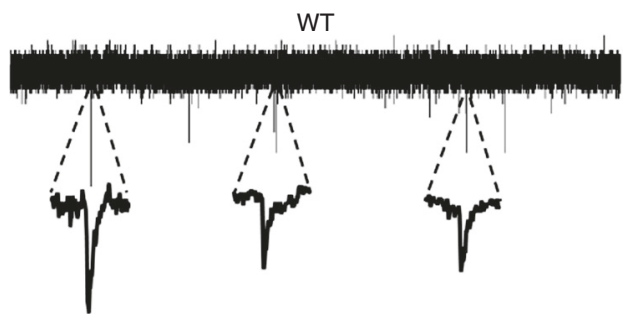

b

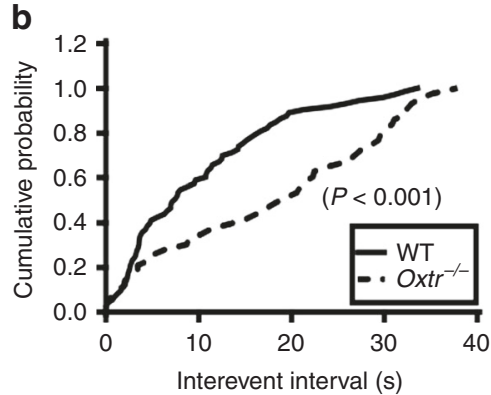

f

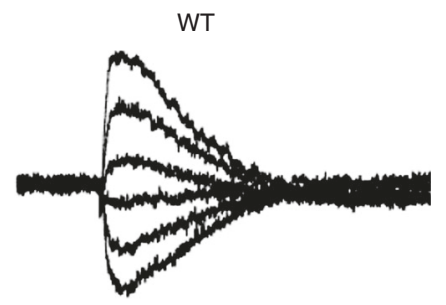

C

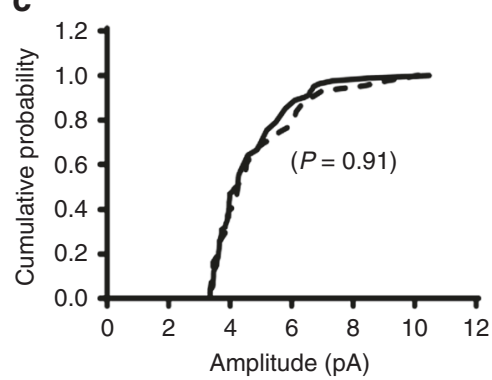

d

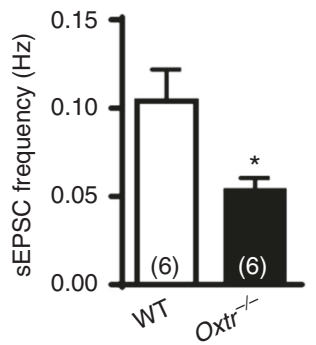

e

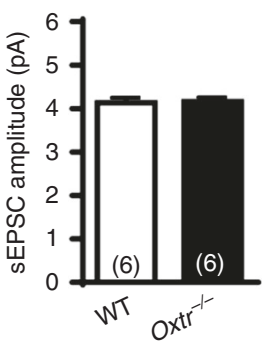

$2 \mathrm{pA} L$

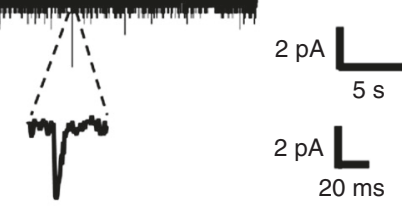

g

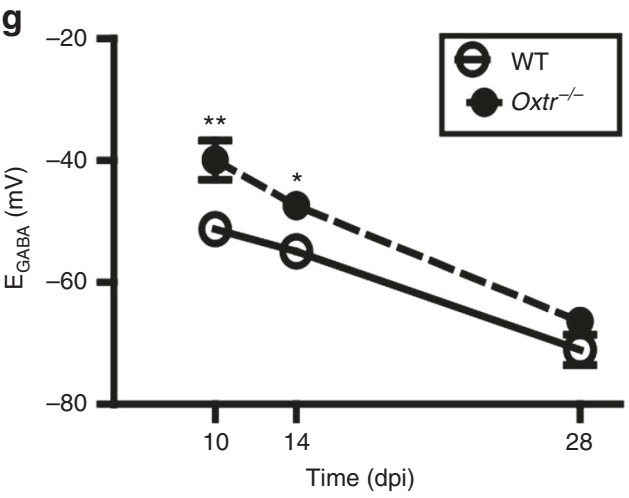

h

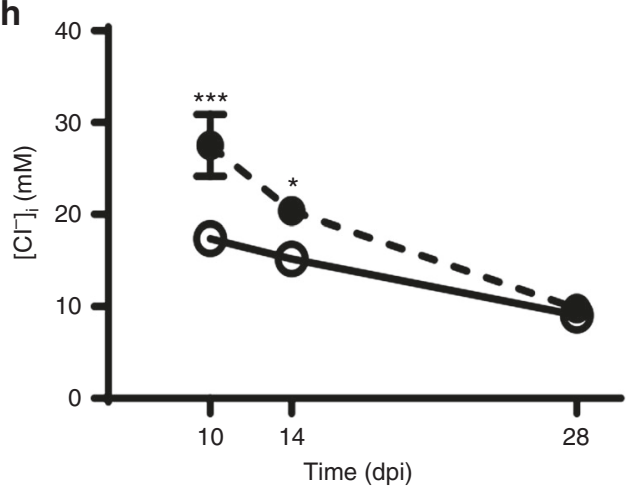

Fig. 5 Effects of Oxtr deletion on excitatory and inhibitory synaptic transmission of newly generated DGCs. a Representative traces of sEPSCs recorded from newly generated DGCs in slices from WT and Oxtr ${ }^{-/-}$mice at 14 dpi. b, c Cumulative probability plots of sEPSC $\mathbf{b}$ interevent intervals and $\mathbf{c}$ amplitude ( $n=6$ neurons from 4 mice per genotype, Kolmogorov-Smirnov test) in newly generated DGCs from WT and Oxtr ${ }^{-/-}$mice at 14 dpi. $\mathbf{d}$, e Summary bar graphs depicting the averaged $\mathbf{d}$ frequency and $\mathbf{e}$ amplitude of sEPSCs in newly generated DGCs from WT and Oxtr ${ }^{-/-}$mice at 14 dpi $(n=6$ neurons from 4 mice per genotype; ${ }^{\star} P<0.05$, unpaired two-tailed Student's $t$ test). $\mathbf{f}$ Left panel, Sample traces of evoked IPSCs recorded at different holding potentials ranging from -70 to $-20 \mathrm{mV}$ (10 mV step) from newly generated DGCs in slices from WT and Oxtr ${ }^{-/-}$mice at 10 dpi. Right panel, current-voltage plot of the peak current of evoked IPSCs obtained from the sample traces. $\mathbf{g}$ Summary data comparing the reversal potential of GABA-mediated IPSCs ( $E_{\mathrm{GABA}}$ ) of newly generated DGCs from WT and Oxtr ${ }^{-/-}$mice at 10,14 , and $28 \mathrm{dpi}\left(n=4-5\right.$ neurons from 4 mice for each group; ${ }^{\star} P<0.05$, ${ }^{\star \star} P<0.01$, two-way ANOVA with Bonferroni's post hoc test). $\mathbf{h}$ Summary data comparing the calculated intracellular chloride concentrations $\left(\left[\mathrm{Cl}^{-}\right]_{\mathrm{i}}\right)$ in newly generated DGCs from WT and $O x^{-1}{ }^{-1}$ mice at 10, 14, and $28 \mathrm{dpi}\left(n=4-5\right.$ neurons from 4 mice for each group; ${ }^{\star} P<0.05$, ${ }^{\star \star \star} P<0.001$, two-way ANOVA with Bonferroni's post hoc test). Data represent the mean \pm s.e.m 
a
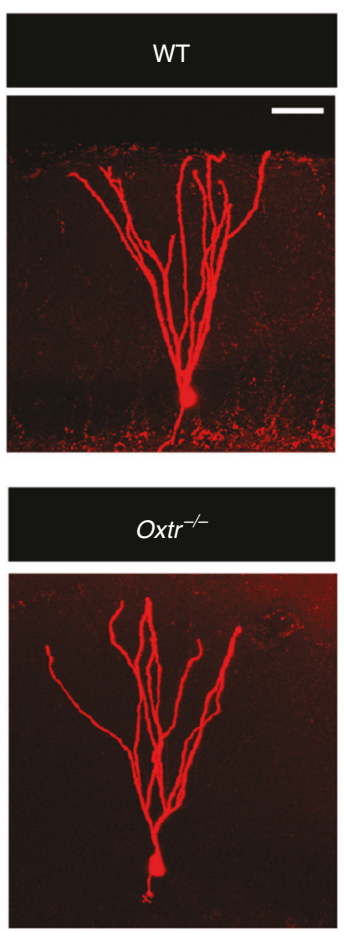

b

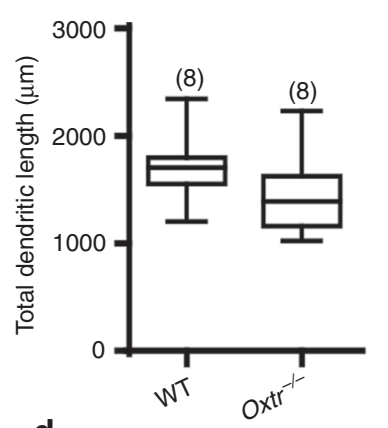

d

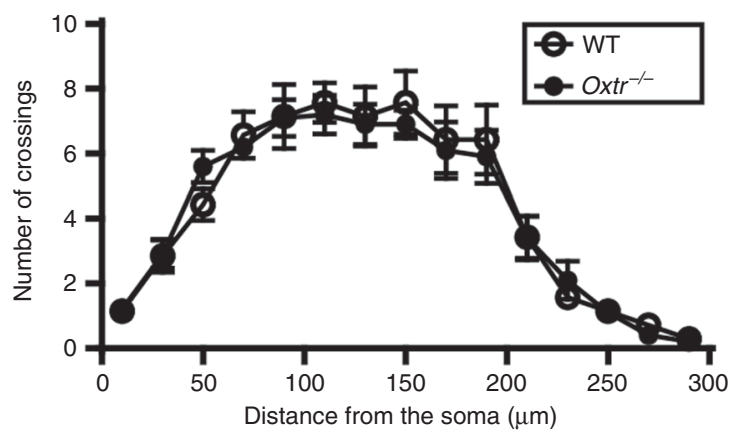

c

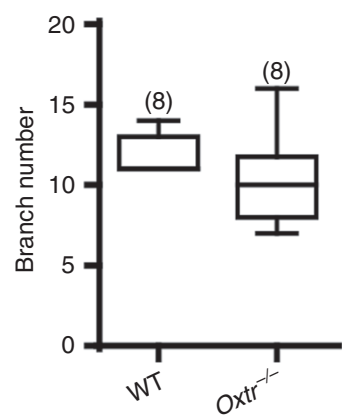

e
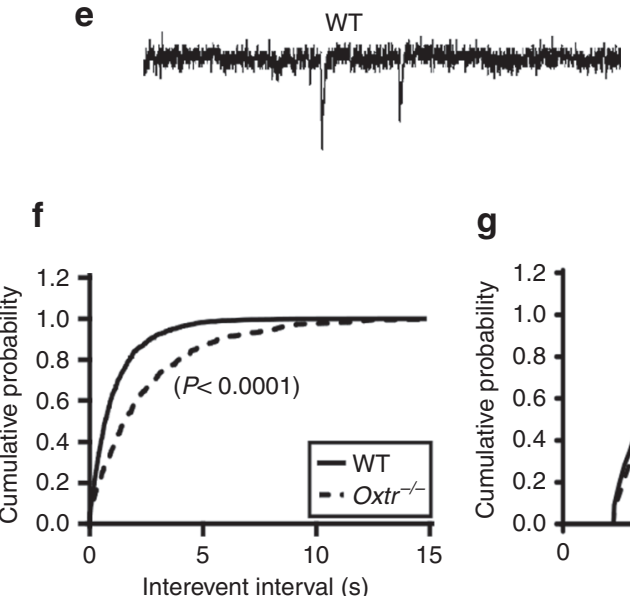

g

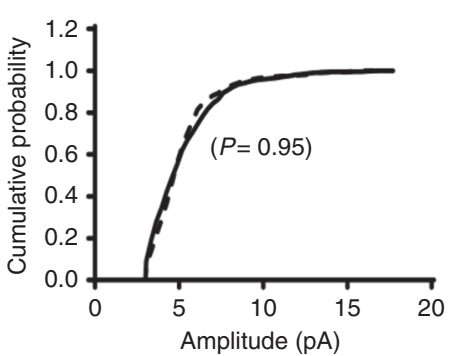

$\mathrm{Oxtr}^{-/-}$

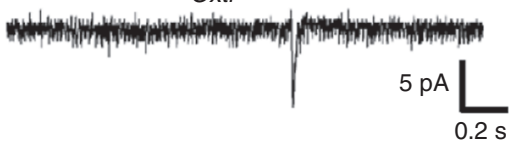

h

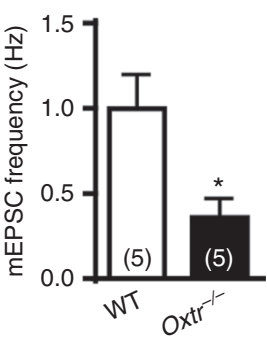

i

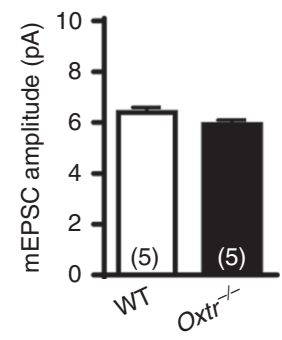

Fig. 6 Effects of Oxtr deletion on morphological features and excitatory synaptic transmission of mature DGCs. a Representative micrographs of biocytinfilled mature DGCs from WT and Oxtr ${ }^{-/-}$mice. Scale bar, $50 \mu \mathrm{m}$. b, c Summary bar graphs depicting $\mathbf{b}$ the total dendritic length and $\mathbf{c}$ branch number of mature DGCs from WT and Oxtr ${ }^{-/-}$mice $(n=8$ neurons from 5 mice per genotype; unpaired two-tailed Student's $t$ test). d Sholl analysis of dendritic complexity of mature DGCs from WT and Oxtr ${ }^{-/-}$mice $(n=8$ neurons from 5 mice per genotype, two-way ANOVA). e Representative traces of mEPSCs recorded from mature DGCs in slices from WT and Oxtr ${ }^{-1-}$ mice. $\mathbf{f}, \mathbf{g}$ Cumulative probability plots of mEPSC $\mathbf{f}$ interevent intervals and $\mathbf{g}$ amplitude $(n=5$ neurons from 3 mice per genotype, Kolmogorov-Smirnov test) in mature DGCs from WT and Oxtr ${ }^{-/-}$mice. $\mathbf{h}$, i Summary bar graphs depicting the averaged $\mathbf{h}$ frequency and $\mathbf{i}$ amplitude of mEPSCs in mature DGCs from WT and Oxtr ${ }^{-/-}$mice $\left(n=5\right.$ neurons from 3 mice per genotype; ${ }^{\star} P<0.05$, unpaired two-tailed Student's $t$ test). Data represent the mean \pm s.e.m

recordings to confirm the effects of $\mathrm{CNO}$ in $\mathrm{AAV}$-infected neurons. Application of $\mathrm{CNO}(1 \mu \mathrm{M})$ significantly decreased spiking responses to $+150 \mathrm{pA}$ square current pulses in hM4Diexpressing $\left(\mathrm{hM}_{4} \mathrm{Di}^{+}\right)$neurons but increased spiking responses in hM3Dq-expressing $\left(\mathrm{hM}^{2} \mathrm{Dq}^{+}\right)$neurons (Fig. 8c). In Oxtr ${ }^{-/}$ mice, we found that the total number of $\mathrm{BrdU}^{+}$cells was significantly increased by hM3Dq/CNO-based approach (Fig. 8d, e). Conversely, hM4Di/CNO-based approach led to a decrease in the total number of $\mathrm{BrdU}^{+}$cells in WT mice (Fig. 8d, e). To evaluate whether chronic $\mathrm{CNO}$ administration may cause cell death of CA3 pyramidal neurons, we used terminal deoxynucleotidyl transferase-mediated dUTP nick-end labeling (TUNEL) assay to assess the extent of apoptotic death. No TUNEL-positive cells were found in the CA3 of WT mice with or without hM3Dq/CNO treatment compared with DNase-treated positive control (Supplementary Fig. 7), confirming that our chronic CNO treatment protocol does not induce apoptotic cell death.

Since Oxtr deletion in $\mathrm{Oxtr}^{-1-}$ mice was not restricted to the CA3, the loss of OXTR function in other hippocampal or forebrain regions may also contribute to impair the survival and maturation of newly generated DGCs. To circumvent this limitation, in a subset of experiments, we used Oxtr/f mice in combination with Cre recombinase-mediated gene deletion in a localized fashion through bilateral stereotaxic injections of AAV- 
Cre-GFP or control AAV-GFP vectors targeting the CA3, under control of the CaMKII $\alpha$ promoter, favoring expression to excitatory neurons. One day before stereotaxic injection of AAV-GFP or AAV-Cre-GFP, mice were subjected to multiple BrdU injections, and the number of $\mathrm{BrdU}^{+}$cells was measured 2 weeks after the last BrdU injection (Supplementary Fig. 8a). The successful transduction of AAV-GFP or AAV-Cre-GFP was confirmed by immunohistochemistry (Supplementary Fig. 8b). Quantitative real-time PCR analysis confirmed the loss of Oxtr mRNA expression in the CA3 2 weeks following the stereotactic injection of AAV-Cre-GFP (Supplementary Fig. 8c). Consistent with the results from $\mathrm{Oxtr}^{-/-}$mice, we found a significant reduction of the total of $\mathrm{BrdU}^{+}$cells in Oxtr f/f mice with AAV-Cre-GFP injections compared with those injected with of a

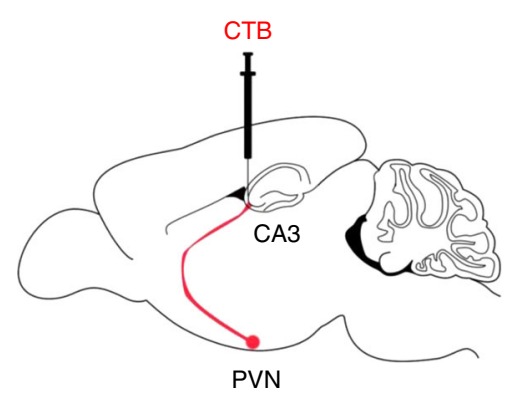

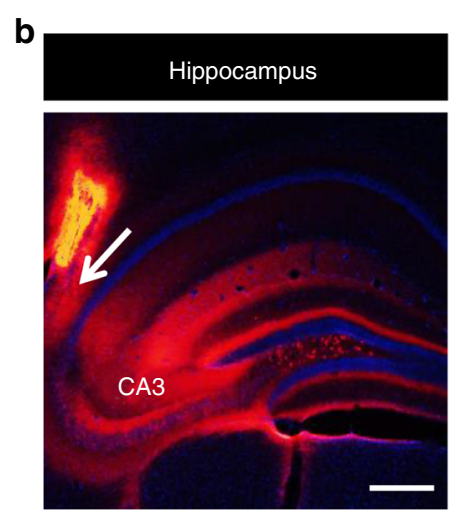
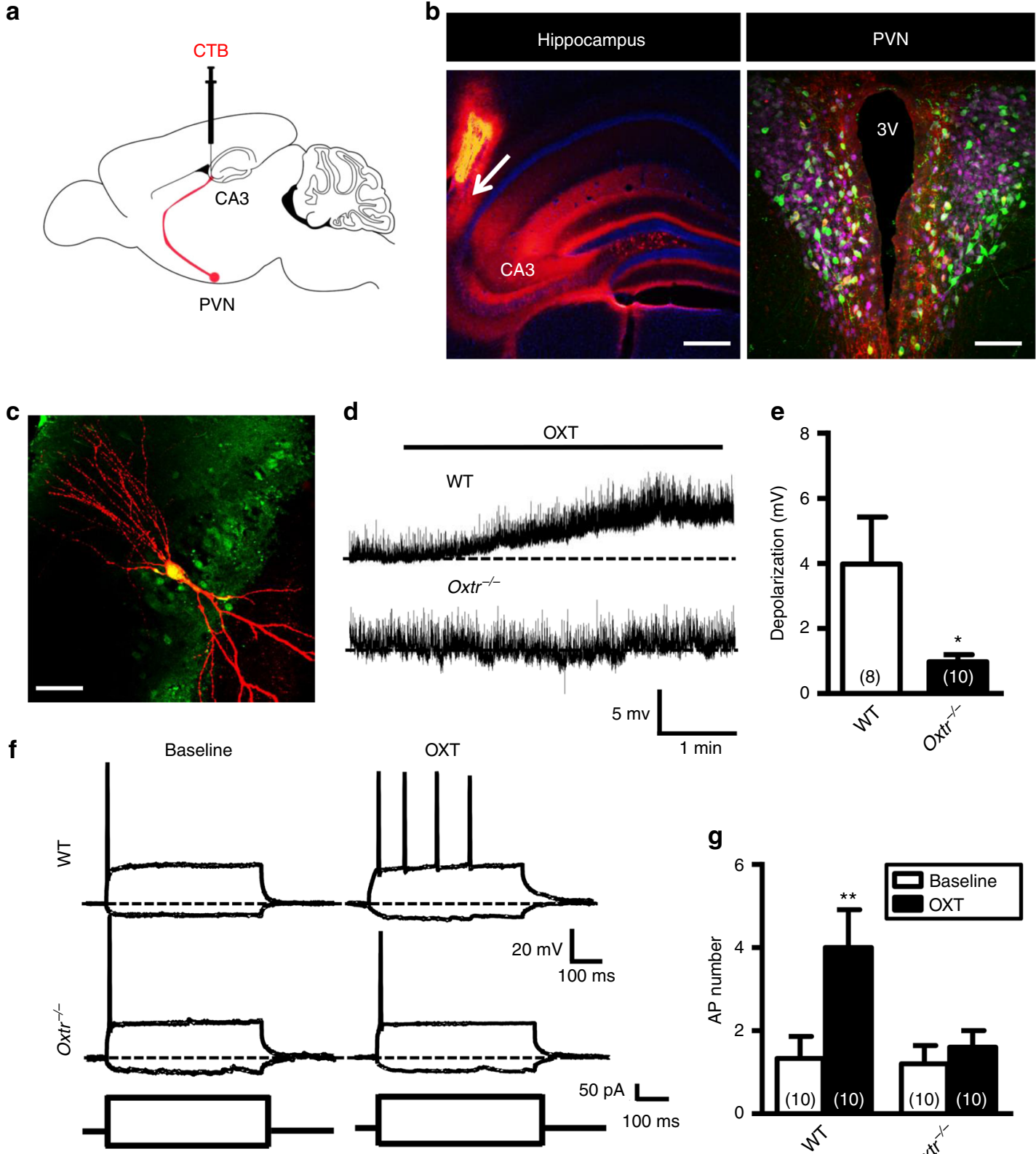

\section{g}

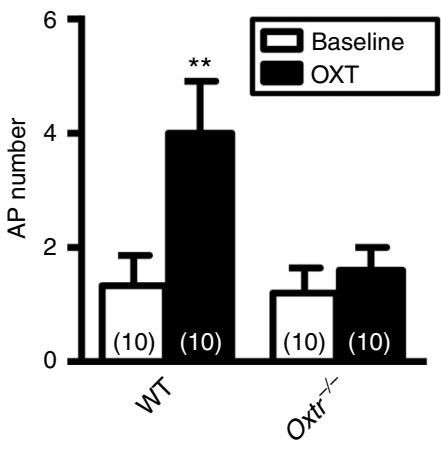

h
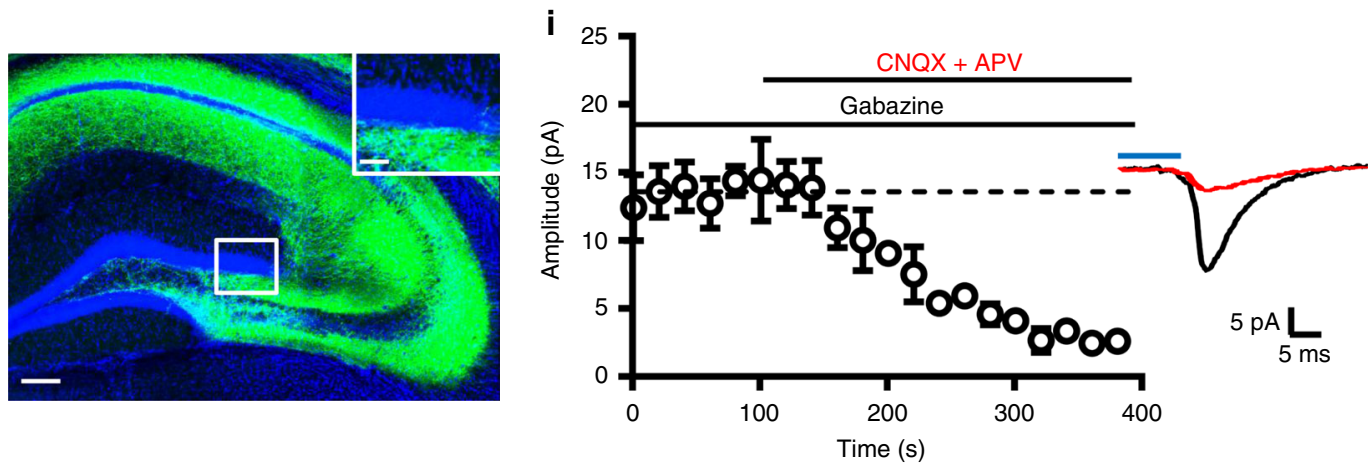
AAV-GFP (Supplementary Fig. 8d, e). We also used retroviral approach to label newly generated DGCs in Oxtr f/f mice with AAV-GFP or AAV-Cre-GFP injections. Engineered retroviruses expressing EGFP were stereotaxically microinjected into the hilus of the DG 1 day before AAV-GFP or AAV-Cre-GFP treatment, and the dendritic arborization of $\mathrm{EGFP}^{+}$DGCs was measured at 14 dpi (Supplementary Fig. 8f). We confirmed a significant reduction in both dendritic length (Supplementary Fig. 8g, h) and branch number (Supplementary Fig. 8g, i) in $\mathrm{EGFP}^{+}$DGCs from Oxtr f/f mice with AAV-Cre-GFP injections compared with those injected with AAV-GFP. Taken together, these results suggest that CA3 neuronal activity plays an important role in regulating adult hippocampal neurogenesis under basal conditions and OXT controls neurogenesis via OXTR expressed in CA3 pyramidal neurons.

\section{Discussion}

In the present study, we examined the role of endogenous OXT signaling in adult hippocampal neurogenesis. There are three major findings. First, OXTR is not expressed in neural progenitor cells reside within the subgranular zone or mature granule cells of adult DG, whereas it is enriched in the CA2 and CA3 of the hippocampus. Second, conditional deletion of Oxtr from hippocampal excitatory neurons leads to impaired survival and maturation of newly generated DGCs. Third, OXT enhances the excitability of hippocampal CA3 pyramidal neurons and CA3 neuronal activity regulates adult hippocampal neurogenesis under basal conditions. Together, these results highlight a non-cell autonomous role for OXT in promoting adult hippocampal neurogenesis via OXTR expressed in CA3 pyramidal neurons.

OXTR is widely expressed in the brain. Early studies using radiolabeled ligands or transgenic approaches have demonstrated abundant expression of OXTR in the hippocampus ${ }^{20,27}$; however, no studies have yet reported whether OXTR is expressed in developing DGCs in adult mouse brain. Using Oxtr $\mathrm{Venus- \textrm {Neo } / +}$ mice in conjunction with immunofluorescent staining for proteins expressed at distinct stages of cell differentiation, we demonstrate here for the first time that OXTR is not expressed in neural progenitor cells or mature granule cells of adult mouse DG. Instead, we confirmed in the previous findings that OXTR is highly expressed in hippocampal CA2 and CA3 pyramidal neurons $^{20}$. We also found that OXTR is present in a subset of calretinin-expressing cells in the hilus of the DG. Given that hilar calretinin-expressing cells in mouse DG could belong to either GABAergic interneurons or mossy cells ${ }^{28}$, additional studies are needed to clarify the neuronal identity of hilar OXTR-expressing cells.

A recent study using pharmacological approaches has proposed that OXT could protect the hippocampus from the detrimental effects of elevated glucocorticoids by promoting adult neurogenesis ${ }^{17}$. It has been demonstrated that both acute peripheral and intra-hippocampal OXT administration enhances cell proliferation, and repeated OXT administration increases the number of newly generated DGCs in adult rat hippocampus ${ }^{17}$. We have extended these findings by underscoring the importance of OXTR signaling in the regulation of adult hippocampal neurogenesis under basal conditions. This view is supported by the observation that conditional deletion of Oxtr from hippocampal excitatory neurons resulted in impaired survival and maturation of newly generated DGCs. Our data suggest, however, that the progenitor cell proliferation and early differentiation into immature neurons were unaltered by conditional deletion of Oxtr. Because we observed no significant changes in cell proliferation in the DG following conditional deletion of Oxtr by an in vivo BrdU incorporation assay, it might be possible that the level of endogenous OXT in the hippocampus is not enough to exert a tonic stimulation of progenitor cell proliferation. Indeed, peripheral OXT administration was previously found to enhance cell proliferation in the adult rat DG in a dose-dependent manner ${ }^{17}$.

Newly generated DGCs exhibit features of dendrites as early as 2 weeks after birth and display overall morphological and functional properties of fully mature GCs at 6 weeks of cell age ${ }^{29,} 30$. In addition, the rate of newly generated DGC maturation highly correlates with the pattern of neuronal activity ${ }^{31}$. We demonstrated that conditional deletion of Oxtr decreased dendritic complexity of newly generated DGCs, as shown by decreased dendritic length and branching, at 14 dpi. However, the suppressive effect of Oxtr deletion on dendrite maturation of newly generated DGCs is short lived and dendritic complexity returns to normal levels at $28 \mathrm{dpi}$. In accordance with morphological observations, our electrophysiological data indicate that the maturation of excitatory synaptic transmission and the developmentally regulated shift in the actions of GABA from excitation to inhibition on newly generated DGCs were delayed by conditional deletion of Oxtr. These data suggest that, although endogenous OXT signaling has effects on morphological properties of newly generated DGCs during their early development, some compensatory mechanisms may exist during late developmental stages to normalize the morphological and physiological changes caused by Oxtr deletion.

A pressing question that follows these observations is how conditional deletion of Oxtr from hippocampal excitatory neurons impairs neurogenesis in the DG. Our results indicate that CA3 pyramidal neurons exert the stimulatory effect of OXT on neurogenesis in the DG of adult mice. By using optogenetic stimulation and immunofluorescence imaging, we found that CA3 pyramidal neurons functionally project to DGCs. In support of our observations, a recent viral-genetic tracing study has provided evidence that newly generated DGCs receives excitatory afferents from CA3 pyramidal neurons ${ }^{32}$. In addition, lesions to the CA3 region of the hippocampus have been found to decrease the survival of newly generated DGCs ${ }^{21}$. Thus, excitatory inputs from

Fig. 7 OXT increases activity of CA3 pyramidal neurons. a A schematic of the approach to label PVN-projecting OXT neurons by injection of CTB-555 into the hippocampal CA3 region and intraperitoneal injection of fluoro-gold ( $4 \% \mathrm{w} / \mathrm{v}$ in $100 \mu \mathrm{l}$ saline). b Left panel, CA3 pyramidal neurons labeled by CTB-555 (red). Section was counterstained with DAPI (blue). Right panel, PVN neurons back-labeled from the hippocampal CA3 region. The CTB ${ }^{+}$cell bodies (red) were found in the caudal portion of the PVN and colocalized OXT (green) and fluoro-gold (pink). The arrow indicates the site of injection. Data were replicated in four mice. Scale bars: left, $250 \mu \mathrm{m}$; right, $100 \mu \mathrm{m}$. c OXTR-containing CA3 pyramidal neuron was targeted for recording, filled with biocytin, and reacted with avidin-rhodamine. Scale bar: $20 \mu \mathrm{m}$. d, e Representative traces and summary data comparing OXT ( $1 \mu \mathrm{M}$ )-induced membrane depolarization in hippocampal CA3 pyramidal neurons from WT and Oxtr ${ }^{-1-}$ mice (WT, $n=8$ neurons from 6 mice; Oxtr ${ }^{-/-}, n=10$ neurons from 6 mice; ${ }^{\star} P<0.05$, unpaired two-tailed Student's $t$ test). $\mathbf{f}, \mathbf{g}$ Representative traces and summary data comparing the effect of OXT ( $1 \mu \mathrm{M}$ ) on action potential firing responses elicited by depolarizing current injection in hippocampal CA3 pyramidal neurons from WT and Oxtr ${ }^{-/-}$mice $(n=10$ neurons from 6 mice per genotype; ${ }^{\star \star} P<0.01$, paired two-tailed Student's $t$ test). $\mathbf{h}$ Representative micrographs showing the expression of ChR2-EYFP in the subgranular zone of the DG and stratum radiatum. Scale bars: 100 and $20 \mu \mathrm{m}$ (rectangle amplification). i Summary graph showing the average amplitude of optically evoked EPSCs recorded in DGCs after application of gabazine $(10 \mu \mathrm{M})$ and CNQX $(20 \mu \mathrm{M})$ plus APV $(50 \mu \mathrm{M})(n=3$ neurons $)$. Data represent the mean \pm s.e.m 
a

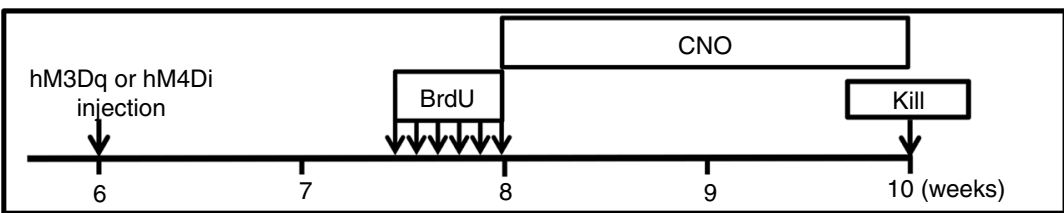

b
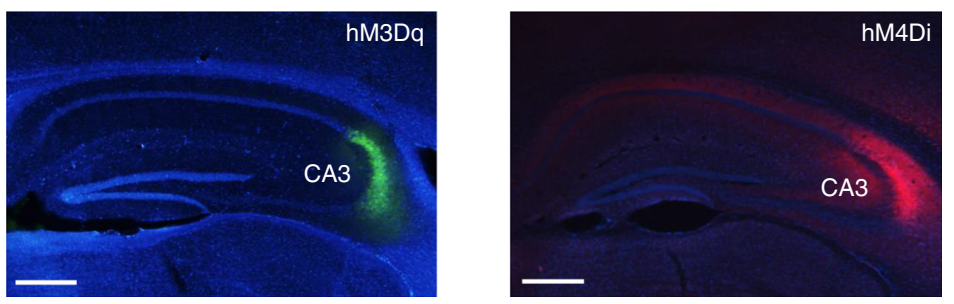

C Control

Vehicle
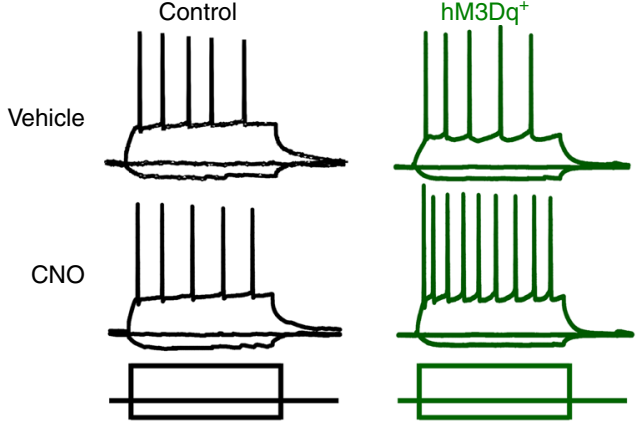

$\mathrm{hM} \mathrm{Di}^{+}$
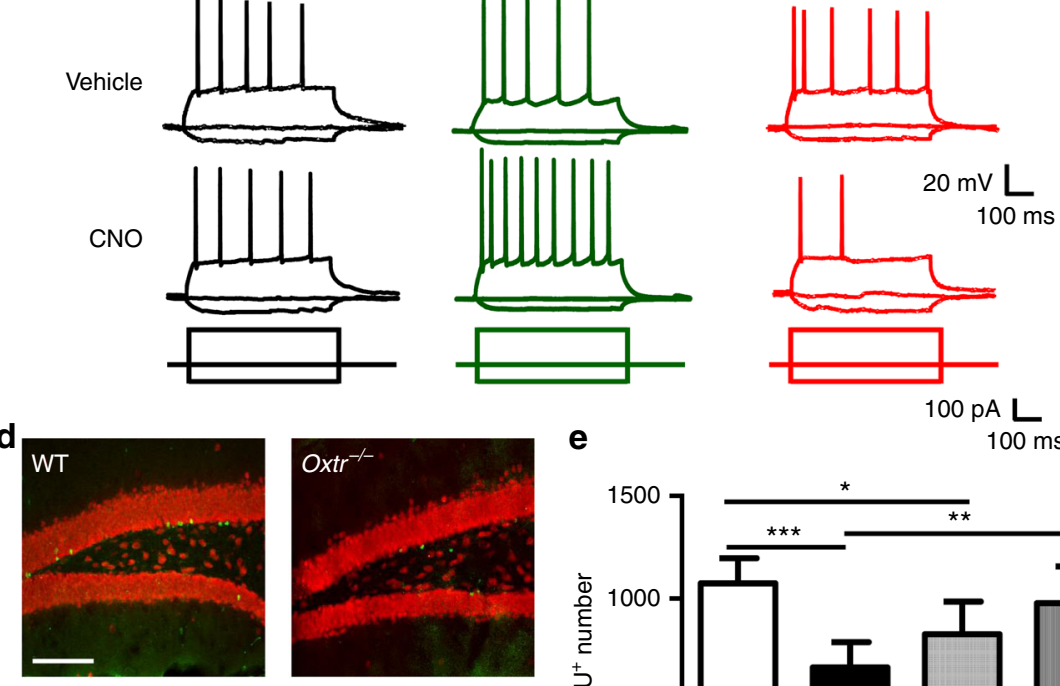

e

$100 \mathrm{~ms}$
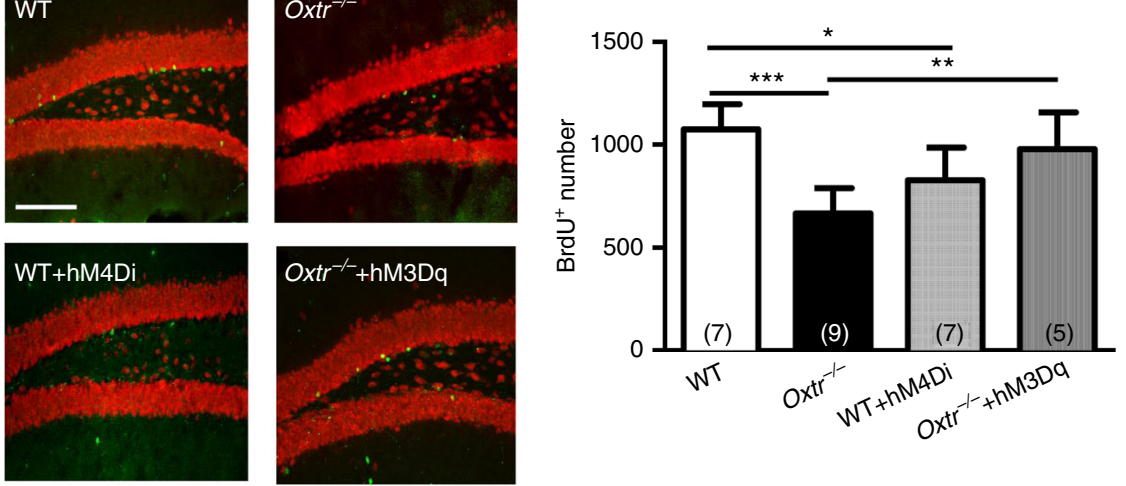

Fig. 8 CA3 pyramidal neuronal activity regulates adult hippocampal neurogenesis. a Schematic representation of the experimental design. One week after stereotaxic injection of AAV-hM3Dq or AAV-hM4Di into the CA3 region, mice were injected six times intraperitoneally with $\mathrm{BrdU}(50 \mathrm{mg} / \mathrm{kg}$ ) at $12 \mathrm{~h}$ intervals. After daily intraperitoneal injection of CNO $(10 \mathrm{mg} / \mathrm{kg})$ for 2 weeks, mice were killed and BrdU incorporation was measured. b Representative micrographs showing the expression of (left panel) hM3Dq and (right panel) hM4Di receptors in the hippocampal CA3 region (counterstained with DAPI, blue). Scale bar: $500 \mu \mathrm{m}$. c Representative traces showing responses of uninfected (control) and infected (hM3Dq ${ }^{+}$or $\mathrm{hM}_{4} \mathrm{Di}^{+}$) neurons to depolarizing current ( + $150 \mathrm{pA}, 500 \mathrm{~ms})$ or hyperpolarizing current pulse $(-50 \mathrm{pA}, 500 \mathrm{~ms})$ under whole-cell current-clamp before and after bath application of CNO $(1 \mu \mathrm{M})$ in the ex vivo hippocampal slices. d Representative immunofluorescence images of hippocampal sections from WT, Oxtr ${ }^{-/-}$, WT + hM4Di and Oxtr $^{-/-}+$hM3Dq stained for BrdU (green) at 14 days after the last BrdU injection (counterstained with NeuN, red). Scale bar, $100 \mu$ m. e Quantification of the total number of BrdU $\mathrm{Bells}^{+}$in the dentate gyrus of WT, Oxtr ${ }^{-/}, \mathrm{WT}+\mathrm{hM} 4 \mathrm{Di}$ and Oxtr ${ }^{-1}+\mathrm{hM} 3 \mathrm{Dq}$ mice at 14 days after BrdU injection $(\mathrm{WT}, \mathrm{n}=7$ mice; Oxtr ${ }^{-/-}, n=9$ mice; WT $+\mathrm{hM} 4 \mathrm{Di}, n=7$ mice; Oxtr ${ }^{-/-}+\mathrm{hM} 3 \mathrm{Dq}, n=5$ mice; ${ }^{\star} P<0.05,{ }^{\star \star} P<0.01$, and ${ }^{\star \star \star} P<0.001$, unpaired two-tailed Student's $t$ test). Data represent the mean \pm s.e.m

CA3 to newly generated DGCs are crucial in maintaining the survival of newly generated DGCs. How do CA3 pyramidal neurons influence the survival of newly generated DGCs? Considering that network activity plays a critical role in the survival of newly generated neurons in the adult brain ${ }^{33-35}$, it is therefore possible that OXT may increase the excitability of CA3 pyramidal neurons, thereby promoting network activity and newly generated DGC survival. In support of this view, we observed that bath application OXT induced a membrane depolarization and increased the number of action potentials in response to postsynaptic depolarizing current injection, suggesting a role for OXT in regulating the excitability of CA3 pyramidal neurons. Our findings that chemogenetic activation of CA3 pyramidal neurons enhanced the survival of newly generated DGCs in Oxtr $-/-$ mice, whereas chemogenetic inhibition of CA3 pyramidal neurons decreased the number of newly generated DGCs in WT mice, clearly confirm that CA3 pyramidal neuron activity can influence the survival of newly generated DGCs.

OXT may exert its biological effects through the activation of OXTR-expressing interneurons. It was previously demonstrated 
that OXT activates a subpopulation of GABAergic interneurons within the lateral subdivision of the central nucleus of the amygdala (CeA) that inhibits activity in medial CeA neurons, thereby attenuating conditioned fear responses 36,37 . Furthermore, OXT has been shown to enhance hippocampal spike transmission by modulating fast-spiking interneurons ${ }^{16}$. Very recent work also reports that OXT can activate a small subset of fast-spiking hilar GABAergic interneurons, which provide local inhibition to mossy cells in the $\mathrm{DG}^{38}$. In this context, our immunofluorescent staining study demonstrates that a small fraction of hilar OXTR-expressing cells are also immunoreactive for GAD67, calretinin, or parvalbumin. It is worth noting that, although parvalbumin interneuron activation has been shown to effectively promote newborn neuronal progeny survival and development via GABA signaling ${ }^{34,} 39$, this mechanism cannot account for the observed impaired survival of newly generated DGCs in our Oxtr conditional knockout mice. Indeed, we conditionally deleted Oxtr under the control of the CaMKII $\alpha$ promotor, which was not expressed in parvalbumin-expressing interneuron $^{40}$. A recent study has demonstrated that CaMKII was expressed in $\sim 40 \%$ calretinin-immunoreactive neurons in the DG of 4- to 5-week-old mice ${ }^{41}$; however, we rarely detected double-labeled neurons immunoreactive for CaMKII $\alpha$ and calretinin in 10-week-old mice (Lin et al., unpublished observation). This may exclude a role of OXTR-expressing calretinin interneurons in mediating the effects of conditional deletion of Oxtr on adult DG neurogenesis. Since Oxtr deletion in conditional Oxtr knockout mice was not restricted to the hippocampus, we could not exclude the possibility that the observed impairment of adult neurogenesis in $\mathrm{Oxtr}^{-/-}$mice may be partially mediated through OXTR-expressing excitatory neurons within extrahippocampal regions that also provide afferent inputs to the $D G$, such as the amygdala ${ }^{2}$. Although we have conducted sitespecific manipulation experiments to confirm the role of hippocampal CA3 OXT signaling in regulating DG neurogenesis, our study cannot definitively rule out the possibility that CA3 pyramidal neurons may, in part, control DG neurogenesis indirectly through the regulation of subsets of hilar GABAergic interneuron activity. Further research is warranted to test this possibility.

In conclusion, we provide compelling evidence that conditional deletion of Oxtr from hippocampal excitatory neurons results in reduced survival and impaired maturation of newly generated DGCs, suggesting a potential role for endogenous OXTR signaling in regulating adult hippocampal neurogenesis. Our results also reveal that CA3 pyramidal neurons play an important role in the regulation of adult hippocampal neurogenesis and OXT controls adult hippocampal neurogenesis through an indirect non-cell autonomous mechanism by OXTR expressed in CA3 pyramidal neurons. Given the prominent role of endogenous OXT signaling in enhancing adult hippocampal neurogenesis, we therefore speculate that it may serve as a potential therapeutic target for neurodegenerative disorders.

\section{Methods}

Animals. Adult male C57BL/6 (8-12 weeks old), homozygous Oxtr-floxed (Oxtr f/f), and CaMKII $\alpha$-Cre transgenic mice were originally obtained from The Jackson Laboratory or and bred within our animal facility. Oxtr/f mice were crossed to CaMKII $\alpha$-Cre mice to generate Oxtr conditional knockout $\left(\mathrm{Oxtr}^{--}\right)$mice in the C57BL/6 genetic background. The heterozygous OxtrVenus-Neo/+ mice were generated as described previously ${ }^{20}$. Mice were genotyped by a PCR-based method using genomic DNA isolated from tail samples. The primers used were as follows: Oxtr, forward (5'-GGCTCAGGCTTTCTCTACTT-3') and reverse (5'-GTTGGG AACAGCGGTGATTA- $3^{\prime}$ ); Cre, forward (5'-GCGGTCTGGCAGTAAAAACT ATC-3') and reverse (5'-GTGAAACAGCATTGCTGTCAACTT-3'); Venus, forward (5'-CTGACCCTGAAGCTGATCT-3') and reverse (5'-GGTAGCTCAGGTA GTGGTTG- $\left.{ }^{\prime}\right)$. Mice were housed in groups of three in a humidity- and temperature-controlled $\left(25 \pm 1{ }^{\circ} \mathrm{C}\right)$ vivarium on a 12-h light/dark cycle with access to food and water ad libitum. All experimental procedures were conducted in accordance with the National Institutes of Health Guide for the Care and Use of Laboratory Animals and were approved by the Institutional Animal Care and Use Committee of National Cheng Kung University. All efforts were made to minimize the number of animals used and their suffering.

Quantitative real-time PCR (qPCR). Total RNA was isolated from hippocampal CA2, CA3, and hypothalamus tissue lysates using a Tri Reagent kit (Molecular Research Center) and treated with RNase-free DNase (RQ1; Promega) to remove potential contamination by genomic DNA. Total RNA $(2 \mu \mathrm{g})$ from samples was reverse transcribed using a SuperScript cDNA synthesis kit (Invitrogen). qPCR was performed on the Roche LightCycler instrument (Roche Diagnostics) using the FastStart DNA Master SYBR Green I kit (Roche Applied Science) according to the manufacturer's instructions. The primers used in this experiment for Oxtr were as follows: forward (5'-TTCTTCGTGCAGATGTGGAG-3') and reverse (5'-CCTTC AGGTACCGAGCAGAG- $3^{\prime}$ ). The PCR reactions were run for 40 cycles. Each amplification cycle included denaturation at $95^{\circ} \mathrm{C}$ for $20 \mathrm{~s}$, annealing at $58^{\circ} \mathrm{C}$ for $20 \mathrm{~s}$, and extension at $72{ }^{\circ} \mathrm{C}$ for $40 \mathrm{~s}$. All reactions were repeated in duplicate and data were analyzed by lightcycler relative quantification software. The expression levels of the target gene were normalized to $\beta$-actin rRNA.

Immunofluorescence. OxtrVenus-Neo/+ mice were deeply anesthetized with sodium pentobarbital $(50 \mathrm{mg} / \mathrm{kg}$, intraperitoneally) and perfused transcardially with PBS and $4 \%$ paraformaldehyde. After the perfusion, brains were removed and continue to fix in $4 \%$ paraformaldehyde for $24 \mathrm{~h}$ at $4{ }^{\circ} \mathrm{C}$ and then transferred to the solution containing $30 \%$ sucrose that immersed in $4{ }^{\circ} \mathrm{C}$ for at least $48 \mathrm{~h}$ before slicing. Coronal slices were sectioned to a $40 \mu \mathrm{m}$ thickness, washed with $0.3 \%$ Triton $\mathrm{X}-100$, and then incubated for blocking with solution containing $3 \%$ goat serum in PBS. After blocking, the sections were incubated in the primary antibodies against nestin (1:500; Millipore, MAB353), Ki67 (1:500; Abcam, ab15580), DCX (1:500; Cell Signaling Technology, \#4604), NeuN (1:1000; Millipore, ABN78), calretinin (1:500; Millipore, MAB1568), parvalbumin (1:500; Swant, PV235), calbindin (1:500; Millipore, AB1778), CaMKII $\alpha$ (1:500; Novas Biologicals, NB100-81830), or GAD67 (1:500, Millipore, MAB5406). Finally, sections were washed with TBS-T (10 mM Tris- $\mathrm{HCl}, 150 \mathrm{mM} \mathrm{NaCl}$, and $0.025 \%$ Tween $20 ; \mathrm{pH} 7.4$ ) and then incubated with the secondary Alexa Fluor 568 antibody (Life Technologies) for $2 \mathrm{~h}$ at room temperature in blocking buffer. The immunostained sections were collected on separate gelatin-subbed glass slides, rinsed extensively in PBS, and mounted with ProLong Gold Antifade Reagent (Invitrogen). Fluorescence images of neurons were obtained using an Olympus FluoView FV1000 confocal microscope with sequential acquisition setting at a resolution of $1024 \times 1024$ pixels, $z$-stack with 15-20 optical sections. All images were imported into NIH ImageJ software (National Institutes of Health) for analysis, and all the parameters used were kept consistent during capturing.

BrdU injection and quantification of newly generated DGCs. For proliferation assay, mice were received single pulse of BrdU (50 mg/kg; Sigma-Aldrich) injection. After $2 \mathrm{~h}$, mice were perfused with $4 \%$ paraformaldehyde in PBS (pH 7.4) and brain tissue collected. For quantification of the survival neurons, mice were injected six times intraperitoneally with BrdU at $12 \mathrm{~h}$ intervals and killed by transcardial perfusion after 2 and 4 weeks after the last BrdU injection. For studying the effect of CA3 neuronal activity on newborn neurons survival, AAV-hM3Dq or AAVhM4Di was bilaterally injected into the CA3 region. One week later, mice were injected six times intraperitoneally with BrdU at $12 \mathrm{~h}$ interval and daily intraperitoneally injected with CNO (10 mg/kg; Sigma-Aldrich) for continuous 2 weeks until killing. The entire dentate gyrus (DG, -0.9 to $-4.2 \mathrm{~mm}$ from bregma) was sectioned coronally at a thickness of $40 \mu \mathrm{m}$ using a sliding microtome (Leica SM2010R).

Fluorescent immunolabelling was used for counting the number of newly generated DGCs. For BrdU/Ki67, BrdU/DCX, or BrdU/NeuN double labeling, free-floating sections were denatured in $10 \mathrm{mM}$ saline-sodium citrate buffer at $85^{\circ} \mathrm{C}$ for $20 \mathrm{~min}$ and then incubated at $37^{\circ} \mathrm{C}$ for $30 \mathrm{~min}$ in $2 \mathrm{~N} \mathrm{HCl}$. Sections were rinsed twice for $5 \mathrm{~min}$ at $25^{\circ} \mathrm{C}$ in $0.1 \mathrm{M} \mathrm{Na}$ borate $(\mathrm{pH} 8.5)$ and then incubated in the primary antibodies against BrdU (1:500; Millipore, MAB4072), Ki67(1:500 Abcam, ab15580), DCX (1:500; Cell Signaling Technology, \#4604), or NeuN (1:1000; Millipore, ABN78) overnight at $4{ }^{\circ} \mathrm{C}$ in PBS with $0.1 \%$ Triton X-100. Finally, sections were washed with PBS and then incubated with the secondary Alexa Fluor 488, 568, or 647 antibodies (Life Technologies) for $2 \mathrm{~h}$ at room temperature. The nuclei were visualized using 4',6-diaminodino-2-phenylindole (DAPI, 1:5000; Sigma-Aldrich) staining. The immunostained sections were collected on separate gelatin-subbed glass slides, rinsed extensively in PBS, and mounted with ProLong Gold Antifade Reagent (Invitrogen).

Quantification of BrdU-labeled cells was performed using a modified unbiased stereology protocol as described previously ${ }^{42,43}$. Every sixth section covering the entire DG was processed for BrdU immunohistochemistry. All BrdU-labeled cells in the granule cell layer, subgranular zone, and hilus were counted under fluorescent illumination at $\times 400$ using an Olympus BX51 microscope coupled to an Olympus DP70 digital camera. Unbiased stereologic analysis was applied to coun BrdU-labeled cells ${ }^{43}$. Total cell numbers were estimated by multiplying the number of cells counted in every sixth section by six. 
Fluorescent in situ hybridization. Fluorescent in situ hybridization was performed using RNAscope ${ }^{\circledast u}$ Multiplex Fluorescent Reagent Kit 2.0 according to the manufacturer's instructions (Advanced Cell Diagnostics). Briefly, brain sections $(16 \mu \mathrm{m})$ were fixed in $4 \%$ paraformaldehyde for $15 \mathrm{~min}$ and dehydrated through graded ethanol solutions $(50,70$, and $100 \%)$ for 5 min each. Sections were subjected to reagent pretreat 3 at $25^{\circ} \mathrm{C}$ for $30 \mathrm{~min}$ and then hybridized with probes at $40^{\circ} \mathrm{C}$ for $2 \mathrm{~h}$ in a humidified oven. The Oxtr-O1 probe (Cat\# 454011) and the CaMKIIL probe (Cat\# 445231) were used to target exon 3 of the Oxtr mRNA and CaMKII $\alpha$ mRNA. After hybridization, brain sections were sequentially applied with a series of probe signal amplification steps, rinsed with ACD wash buffer twice for 2 min between each step and finally counterstained with DAPI and mounted with VECTASHIELD antifade mounting medium (Vector Laboratories) containing DAPI (1:5000; Sigma-Aldrich).

\section{Retrovirus production, stereotaxic injection, and analysis. Engineered} self-inactivating murine retroviruses expressing EGFP were used to label proliferating cells and their progeny in the DG of adult mice as described previously $y^{43}, 45$. High titers of engineered retroviruses $\left(1 \times 10^{9} \mathrm{unit} / \mathrm{ml}\right)$ were produced by co-transfection of retroviral vectors and VSV-G into HEK293GP cells followed by ultracentrifugation of viral supernatant. The Cre recombinase (Addgene, plasmid \#20781) was cloned into a retroviral expression vector Ubi-X-2A-EGFP.

Adult male WT or homozygous $\mathrm{Oxtr}^{-/-}$mice were anesthetized and the purified retroviruses were stereotaxically injected into the DG at 4 sites $(0.5 \mu \mathrm{l} /$ site at $0.25 \mu \mathrm{l} / \mathrm{min}$ ) with the following coordinates (anterior-posterior $=-2 \mathrm{~mm}$ from bregma, lateral $\pm 1.6 \mathrm{~mm}$, ventral $=2.5 \mathrm{~mm}$; anterior-posterior $3 \mathrm{~mm}$ from bregma, lateral $= \pm 2.6 \mathrm{~mm}$, ventral $=3.2 \mathrm{~mm}$ ) in accordance with the description by Franklin and Paxinos ${ }^{46}$. Mice were killed at 14 and 28 dpi for morphological analysis. Coronal brain sections ( $40 \mu \mathrm{m}$ thick) were prepared from viral-injected mice and processed for immunostaining as previously described ${ }^{42,44}$. Sections were incubated for $30 \mathrm{~min}$ in DAPI before washing and mounting. Images were acquired on an Olympus FluoView FV1200MPE multiphoton confocal system using a multi-track configuration. Z-series stack of confocal images were taken and a single confocal image slice with the largest soma area for individual $\mathrm{EGFP}^{+}$neurons was chosen for quantification using NIH ImageJ program.

For dendritic development analysis, three-dimensional reconstruction of the entire processes of each neuron was made from Z-series stacks of confocal images. The projection images were semi-automatically traced with NIH ImageJ (http://rsb. info.nih.gov/ij/) using the NeuronJ Plugin. All EGFP ${ }^{+}$DGCs with intact dendritic trees were analyzed for total dendritic length and branch number. Dendritic complexity was quantified by Sholl analysis by counting the number of dendrites that crossed a series of concentric circles at $10 \mu \mathrm{m}$ intervals from the cell soma using the Sholl analysis plug-in.

Adeno-associated virus production. DNA plasmids encoding pAAV-CaMKII $\alpha$ hM3Dq-internal ribosomal entry site (IRES)-mCitrine (Addgene, plasmid \#50466), pAAV-CaMKII $\alpha$-hM4Di-mCherry (Addgene, plasmid \#50477), pAAV-CaMKII $\alpha$ hChR2(H134R)-EYFP (Addgene, plasmid \#26969), pAAV-CaMKII $\alpha$-EGFP (Addgene, plasmid \#50469), and pAAV-Ubi-eGFP (Addgene, plasmid \#62518) were obtained from Addgene. pAAV-CaMKII $\alpha$-Cre-GFP was constructed from Cre-GFP empty vector (Addgene, plasmid \#20781) into pAAV-CaMKII $\alpha$-hChR2EYFP (Addgene, plasmid \#26969). Plasmid DNA was amplified, purified, and collected using a standard plasmid maxiprep kit (Qiagen). The purified plasmids were mixed into $\mathrm{CaCl}_{2}$ solution with the DNA plasmid coding AAV-DJ and co-transfected into HEK293T cells using calcium phosphate precipitation methods. Transfected cells were harvested at $72 \mathrm{~h}$ after transfection and the virus was purified using the AAV purification mega kit (Cell Biolabs, Inc.). Viral titers were $5 \times$ $10^{12}$ particles $/ \mathrm{ml}$ and stored in aliquots at $-80^{\circ} \mathrm{C}$ until use.

Retrograde tracing. For retrograde tracing of projecting neurons, fluorescence Alexa Fluor 555-conjugated CTB $(0.1 \mu$; Thermo Fisher, Cat\#C-34776) was injected bilaterally into the CA3 region rostral $-2.5 \mathrm{~mm}$, lateral $\pm 2.8 \mathrm{~mm}$, and ventral $-2.5 \mathrm{~mm}$ at $0.1 \mu \mathrm{l} / \mathrm{min}$ by using $1 \mu \mathrm{l}$ Hamilton syringe. The syringe was slowly retracted after additional 5 min solution diffusion. For double-labeling neuroendocrine neurons, mice were simultaneously received an intraperitoneally injection of fluoro-gold ( $4 \% \mathrm{w} / \mathrm{v}$ in $100 \mu \mathrm{l}$ saline; Santa Cruz, Cat\#SC-358883). Ten days later, the animals were perfused transcardially with PBS followed by $4 \%$ paraformaldehyde solution. Brains were then removed and cut into $40 \mu \mathrm{m}$-thick coronal sections with a Vibrotome and immunofluorescence stained with the primary antibody against OXT (1:500; Millipore, AB911) and fluoro-gold (1:1000; Millipore, AB153-I).

TUNEL assay. Apoptosis examination was performed using in situ cell death detection kit (Roche, Cat\#1684795) following the manufacturer's instruction. Briefly, PFA-perfused brain sections were microwave treated in $0.1 \mathrm{M}$ citrate buffer for $1 \mathrm{~min}$ and washed twice in PBS. For labeling, sections were incubated in TUNEL enzyme solution for $1 \mathrm{~h}$ at $37^{\circ} \mathrm{C}$ in the dark and counterstained with DAPI. Positive controls were obtained by incubating hippocampal slices with DNase $(3000 \mathrm{U} / \mathrm{ml}$ in $50 \mathrm{mM}$ Tris- $\mathrm{HCl}, \mathrm{pH} 7.5,1 \mathrm{mg} / \mathrm{ml} \mathrm{BSA})$ for $15 \mathrm{~min}$ at $25^{\circ} \mathrm{C}$ to induce DNA-strand breaks, prior to labeling procedure.
Electrophysiological recordings. Hippocampal slices were prepared using standard procedures as described previously ${ }^{43}$, 45 . In brief, were anesthetized with isoflurane and decapitated, and brains were rapidly removed and placed in ice-cold oxygenated sucrose cutting solution (containing (in $\mathrm{mM}$ ): sucrose $234, \mathrm{KCl} 2.5$, $\mathrm{CaCl}_{2} 0.5, \mathrm{MgCl}_{2} 7, \mathrm{NaHCO}_{3} 25, \mathrm{NaH}_{2} \mathrm{PO}_{4} 1.25$, and glucose 11 at $\mathrm{pH} 7.3-7.4$ and equilibrated with $\left.95 \% \mathrm{O}_{2}-5 \% \mathrm{CO}_{2}\right)$. Hippocampal slices $(250 \mu \mathrm{m})$ were prepared using a vibrating microtome (VT1200S; Leica) and transferred to a holding chamber of artificial cerebrospinal fluid (ACSF) (containing (in $\mathrm{mM}$ ): $\mathrm{NaCl} 117$, $\mathrm{KCl} 4.7, \mathrm{CaCl}_{2} 2.5, \mathrm{MgCl}_{2} 1.2, \mathrm{NaHCO}_{3} 25, \mathrm{NaH}_{2} \mathrm{PO}_{4} 1.2$, and glucose 11 at $\mathrm{pH}$ 7.3-7.4 and equilibrated with $95 \% \mathrm{O}_{2}-5 \% \mathrm{CO}_{2}$ ) and maintained at room temperature for at least $1 \mathrm{~h}$ before use.

For recording, slices were transferred to a submersion-type recording chamber and fixed at the glass bottom of the chamber with a nylon grid on a platinum frame. The chamber was constantly perfused with ACSF at $32.0 \pm 0.5^{\circ} \mathrm{C}$ with a rate of 2-3 ml/min. Conventional whole-cell and gramicidin-perforated patch-clamp recordings were made from newly generated $\left(\mathrm{EGFP}^{+}\right) \mathrm{DGCs}$, mature $\left(\mathrm{EGFP}^{-}\right)$ DGCs or CA3 pyramidal neurons by using a patch-clamp amplifier (Axopatch 200B, Molecular Devices) under infrared differential interference contrast microscope. Mature DGCs were recorded from the outer portion of the granule cell layer. Data acquisition and analysis were performed using a digitizer (Digidata 1440 A, Molecular Devices) and pCLAMP 9 software (Molecular Devices). Synaptic responses were evoked using a bipolar stainless steel stimulating electrode placed in the middle molecular layer $\sim 100 \mu \mathrm{m}$ away from the recorded cell. For measurement of $E_{G A B A}$ in newly generated DGCs, pharmacologically isolated $\mathrm{GABA}_{\mathrm{A}}$ receptor-mediated IPSCs were recorded using gramicidin-perforated patch under voltage-clamp at different holding potentials in the presence of CNQX $(20 \mu \mathrm{M}$; Tocris Bioscience) and APV (50 $\mu \mathrm{M}$; Tocris Bioscience) to eliminate glutamate currents. A stock solution of gramicidin $(10 \mathrm{mg} / \mathrm{ml})$ was prepared in dimethyl sulfoxide and was then diluted in the pipette solution (containing (in $\mathrm{mM}$ ): $\mathrm{CsCl}$ 135, $\mathrm{MgCl}_{2}$ 2, EGTA 0.5, HEPES 10, pH 7.3, pH 7.3, 290-295 mOsm) to a final concentration of $25 \mu \mathrm{g} / \mathrm{ml}$. After gigaseal formation, the progress of perforation was continuously monitored until the series resistance had stabilized to $\leq 30 \mathrm{M} \Omega$. The intracellular chloride concentration was calculated according to the Nernst equation: $\left[\mathrm{Cl}^{-}\right]_{\mathrm{i}}=\left[\mathrm{Cl}^{-}\right]_{\mathrm{o}} \mathrm{e}\left(\mathrm{E}_{\mathrm{GABA}} \mathrm{F} / \mathrm{RT}\right)$, where the extracellular $\mathrm{Cl}^{-}$concentration $\left[\mathrm{Cl}^{-}\right]_{\mathrm{o}}$ is $125.4 \mathrm{mM}$.

sEPSCs and mEPSCs were recorded from newly generated or mature DGCs held under voltage-clamp at $-70 \mathrm{mV}$ in the presence of gabazine $(10 \mu \mathrm{M}$; Tocris Bioscience) and analyzed off-line using commercially available software (Mini Analysis 4.3; Synaptosoft). Recordings of mEPSCs were conducted in the presence of TTX $(0.5 \mu \mathrm{M}$; Tocris Bioscience). The composition of intracellular solution was (mM): K-gluconate 120, $\mathrm{KCl} 15$, HEPES $10, \mathrm{MgCl}_{2}$ 4, EGTA 0.1, $\mathrm{Na}_{2} \mathrm{ATP} 4$, $\mathrm{Na}_{3}$ GTP 0.3 , phosphocreatine 7 , and $0.5 \% \mathrm{w} / \mathrm{v}$ biocytin, $280-290 \mathrm{mOsm}$ without biocytin, pH 7.3 with $\mathrm{KOH}$. The detection threshold for sEPSCs and mEPSCs was set at $3 \mathrm{pA}$.

To examine the effects of OXT on intrinsic membrane properties of OXTR expressing CA3 pyramidal neurons, hippocampal slices were prepared from OXTR-Venus knock-in mice. After a stable recording of membrane potential under current-clamp condition, OXT $(1 \mu \mathrm{M})$ was applied to the recorded cell by a 5 -min bath application. In some experiments, hyperpolarizing $(-50 \mathrm{pA})$ and depolarizing current pulses $(100 \mathrm{pA})$ of $500 \mathrm{~ms}$ were injected through the recording pipette in current-clamp mode to measure neuronal excitability. The number of action potentials triggered was counted. Biocytin was routinely included in the intracellular solution to allow post hoc staining of the recorded neurons. OXT and L-371257 were purchased from Tocris Bioscience.

To confirm the expression of engineered hM3Dq or hM4Di receptors in CA3 neurons, a depolarizing current pulse $(150 \mathrm{pA}, 500 \mathrm{~ms})$ was injected into the control, $\mathrm{hM}_{3} \mathrm{Dq}^{+}$or $\mathrm{hM}_{4} \mathrm{Di}^{+}$(hM3Dq or hM4Di expression) neurons to induce spiking. Following $10 \mathrm{~min}$ continuous recordings, CNO (1 $\mu \mathrm{M}$; Sigma-Aldrich) was applied into the ACSF and a second depolarizing current $(+150 \mathrm{pA}, 500 \mathrm{~ms})$ or hyperpolarizing current pulse $(-50 \mathrm{pA}, 500 \mathrm{~ms})$ was injected into the $\mathrm{hM}^{\mathrm{D}} \mathrm{Dq}^{-}$, $\mathrm{hM} 3 \mathrm{Dq}^{+}, \mathrm{hM}_{4 \mathrm{Di}^{-}}$, and $\mathrm{hM} 4 \mathrm{Di}^{+}$neurons to induce spiking in order to compare spiking before and after $\mathrm{CNO}$ application.

For in vitro optical stimulation, a multimode optical fiber with core diameter of $200 \mu \mathrm{m}$ (Thorlabs), coupled to a diode-pumped solid-state laser of specific wavelength (473 nm blue laser; Laserglow Technologies), was used. The ending power on brain slices was $\sim 5 \mathrm{~mW} / \mathrm{mm}^{2}$ and synaptic responses were recorded at $-70 \mathrm{mV}$ in voltage-clamp mode.

Statistical analysis. No statistical tests were used to predetermine sample sizes, but our sample sizes were consistent with the previous publication using similar approaches $^{22,43}$. Mice were randomly assigned to viral injection experiments, and investigators were blinded to the group allocation while performing cell number counting, morphological analysis, and electrophysiological recordings. The results are presented as mean \pm s.e.m. All statistical analyses were performed using the Prism 6 software package (GraphPad software). The significance of any difference between two groups was calculated using the paired or unpaired two-tailed Student's $t$ test. One-way ANOVA or repeated measure two-way ANOVA tests were used for multiple groups' comparison and Bonferroni's post hoc analyses were used to assess the significance between isolated groups. Kolmogorov-Smirnov test were 
used in comparison of cumulative frequency distribution. $N$ represents the number of animals used. Values of $P<0.05$ were considered significant.

Data availability. The authors declare that all data supporting the findings of this study are available within the article and its Supplementary Information File.

Received: 16 October 2016 Accepted: 19 July 2017

Published online: 14 September 2017

\section{References}

1. Zimmerman, E. A., Nilaver, G., Hou-Yu, A. \& Silverman, A. J. Vasopressinergic and oxytocinergic pathways in the central nervous system. Fed. Proc 43, 91-96 (1984).

2. Gimpl, G. \& Fahrenholz, F. The oxytocin receptor system: structure, function, and regulation. Physiol. Rev. 81, 629-683 (2001).

3. Buijs, R. M. \& Swaab, D. F. Immuno-electron microscopical demonstration of vasopressin and oxytocin synapses in the limbic system of the rat. Cell Tissue Res. 204, 355-365 (1979).

4. Sofroniew, M. V. Morphology of vasopressin and oxytocin neurons and their central and vascular projections. Prog. Brain Res. 60, 101-114 (1983).

5. Young, W. S. 3rd \& Gainer, H. Transgenesis and the study of expression, cellular targeting and function of oxytocin, vasopressin and their receptors. Neuroendocrinology 78, 185-203 (2003).

6. Meyer-Lindenberg, A., Domes, G., Kirsch, P. \& Heinrichs, M. Oxytocin and vasopressin in the human brain: social neuropeptides for translational medicine. Nat. Rev. Neurosci. 12, 524-538 (2011).

7. Grinevich, V., Knobloch-Bollmann, H. S., Eliava, M., Busnelli, M. \& Chini, B. Assembling the puzzle: pathways of oxytocin signaling in the brain. Biol. Psychiatry 79, 155-164 (2016).

8. Pedersen, C. A. \& Prange, A. J. Jr. Induction of maternal behavior in virgin rats after intracerebroventricular administration of oxytocin. Proc. Natl Acad. Sci. USA 76, 6661-6665 (1979).

9. Nishimori, K. et al. Oxytocin is required for nursing but is not essential for parturition or reproductive behavior. Proc. Natl Acad. Sci. USA 93, 11699-11704 (1996).

10. Neumann, I. D. Brain oxytocin: a key regulator of emotional and social behaviors in both females and males. J. Neuroendocrinol. 20, 858-865 (2008).

11. Neumann, I. D. \& Landgraf, R. Advances in vasopressin and oxytocin-from genes to behavior to disease. Preface Prog. Brain Res. 170, xi-xiii (2008).

12. Lee, H. J., Macbeth, A. H., Pagani, J. H. \& Young, W. S. 3rd. Oxytocin: the great facilitator of life. Prog. Neurobiol. 88, 127-151 (2009).

13. Cohen, H. et al. Hippocampal microinfusion of oxytocin attenuates the behavioural response to stress by means of dynamic interplay with the glucocorticoid-catecholamine responses. J. Neuroendocrinol. 22, 889-904 (2010).

14. Tomizawa, K. et al. Oxytocin improves long-lasting spatial memory during motherhood through MAP kinase cascade. Nat. Neurosci. 6, 384-390 (2003).

15. Lin, Y. T., Huang, C. C. \& Hsu, K. S. Oxytocin promotes long-term potentiation by enhancing epidermal growth factor receptor-mediated local translation of protein kinase Mל. J. Neurosci. 32, 15476-15488 (2012).

16. Owen, S. F. et al. Oxytocin enhances hippocampal spike transmission by modulating fast-spiking interneurons. Nature 500, 458-462 (2013).

17. Leuner, B., Caponiti, J. M. \& Gould, E. Oxytocin stimulates adult neurogenesis even under conditions of stress and elevated glucocorticoids. Hippocampus 22, 861-868 (2012).

18. Zhao, C., Deng, W. \& Gage, F. H. Mechanisms and functional implications of adult neurogenesis. Cell 132, 645-660 (2008).

19. Christian, K. M., Song, H. \& Ming, G. L. Functions and dysfunctions of adult hippocampal neurogenesis. Annu. Rev. Neurosci. 37, 243-262 (2014).

20. Yoshida, M. et al. Evidence that oxytocin exerts anxiolytic effects via oxytocin receptor expressed in serotonergic neurons in mice. J. Neurosci. 29, 2259-2271 (2009).

21. Liu, J. X., Pinnock, S. B. \& Herbert, J. Novel control by the CA3 region of the hippocampus on neurogenesis in the dentate gyrus of the adult rat. PLoS ONE 6, e17562 (2011).

22. Duan, X. et al. Disrupted-in-schizophrenia 1 regulates integration of newly generated neurons in the adult brain. Cell 130, 1146-1158 (2007).

23. Ge, S. et al. GABA regulates synaptic integration of newly generated neurons in the adult brain. Nature 439, 589-593 (2006).

24. Luther, J. A. et al. Neurosecretory and non-neurosecretory parvocellular neurones of the hypothalamic paraventricular nucleus express distinct electrophysiological properties. J. Neuroendocrinol. 14, 929-932 (2002).

25. Liu, X. B. \& Jones, E. G. Localization of alpha type II calcium calmodulindependent protein kinase at glutamatergic but not $\gamma$-aminobutyric acid (GABAergic) synapses in thalamus and cerebral cortex. Proc. Natl Acad. Sci. USA 93, 7332-7336 (1996).
26. Armbruster, B. N., Li, X., Pausch, M. H., Herlitze, S. \& Roth, B. L. Evolving the lock to fit the key to create a family of $\mathrm{G}$ protein-coupled receptors potently activated by an inert ligand. Proc. Natl Acad. Sci. USA 104, 5163-5168 (2007).

27. Elands, J., Beetsma, A., Barberis, C. \& de Kloet, E. R. Topography of the oxytocin receptor system in rat brain: an autoradiographical study with a selective radioiodinated oxytocin antagonist. J. Chem. Neuroanat. 1, 293-302 (1988).

28. Tóth, K. \& Maglóczky, Z. The vulnerability of calretinin-containing hippocampal interneurons to temporal lobe epilepsy. Front. Neuroanat 8, 100 (2014).

29. Espósito, M. S. et al. Neuronal differentiation in the adult hippocampus recapitulates embryonic development. J. Neurosci. 25, 10074-10086 (2005).

30. Zhao, C., Teng, E. M., Summers, R. G. Jr, Ming, G. L. \& Gage, F. H. Distinct morphological stages of dentate granule neuron maturation in the adult mouse hippocampus. J. Neurosci. 26, 3-11 (2006).

31. Piatti, V. C. et al. The timing for neuronal maturation in the adult hippocampus is modulated by local network activity. J. Neurosci. 31, 7715-7728 (2011).

32. Vivar, C. et al. Monosynaptic inputs to new neurons in the dentate gyrus. Nat. Commun. 3, 1107 (2012)

33. Mouret, A. et al. Learning and survival of newly generated neurons: when time matters. J. Neurosci. 28, 11511-11516 (2008).

34. Song, J. et al. Neuronal circuitry mechanism regulating adult quiescent neural stem-cell fate decision. Nature 489, 150-154 (2012).

35. Lepousez, G., Nissant, A. \& Lledo, P. M. Adult neurogenesis and the future of the rejuvenating brain circuits. Neuron 86, 387-401 (2015).

36. Viviani, D. et al. Oxytocin selectively gates fear responses through distinct outputs from the central amygdala. Science 333, 104-107 (2011).

37. Knobloch, H. S. et al. Evoked axonal oxytocin release in the central amygdala attenuates fear response. Neuron 73, 553-566 (2012).

38. Harden, S. W. \& Frazier, C. J. Oxytocin depolarizes fast-spiking hilar interneurons and induces GABA release onto mossy cells of the rat dentate gyrus. Hippocampus 26, 1124-1139 (2016)

39. Song, J. et al. Parvalbumin interneurons mediate neuronal circuitryneurogenesis coupling in the adult hippocampus. Nat. Neurosci. 16, 1728-1730 (2013).

40. Sík, A., Hájos, N., Gulácsi, A., Mody, I. \& Freund, T. F. The absence of a major $\mathrm{Ca}^{2+}$ signaling pathway in GABAergic neurons of the hippocampus. Proc. Natl Acad. Sci. USA 95, 3245-3250 (1998).

41. Arruda-Carvalho, M. et al. Conditional deletion of $\alpha$-CaMKII impairs integration of adult-generated granule cells into dentate gyrus circuits and hippocampus-dependent learning. J. Neurosci. 34, 11919-11928 (2014).

42. West, M. J., Slomianka, L. \& Gundersen, H. J. Unbiased stereological estimation of the total number of neurons in thesubdivisions of the rat hippocampus using the optical fractionator. Anat. Rec. 231, 482-497 (1991).

43. Chen, C. C., Huang, C. C. \& Hsu, K. S. Chronic social stress affects synaptic maturation of newly generated neurons in the adult mouse dentate gyrus. Int. J. Neuropsychopharmacol. 19, pyv097 (2015).

44. Gundersen, H. J., Jensen, E. B., Kiêu, K. \& Nielsen, J. The efficiency of systematic sampling in stereology-reconsidered. J. Microsc. 193, 199-211 (1999).

45. Ge, S., Yang, C. H., Hsu, K. S., Ming, G. L. \& Song, H. A critical period for enhanced synaptic plasticity in newly generated neurons of the adult brain. Neuron 54, 559-566 (2007).

46. Franklin, K. \& Paxinos, G. The Mouse Brain In Stereotaxic Coordinates (Elsevier Academic Press, 2008).

\section{Acknowledgements}

This work was supported by research grants from the National Health Research Institute (NHRI-EX105-10336NI and NHRI-EX106-10613NI), the Ministry of Science and Technology (104-2321-B-006-28, 104-2320-B-006-040-MY3, and 105-2321-B-006-017), and the Ministry of Education (Aim for the Top University Project to the NCKU) of Taiwan (to K.S.H.) and the Integrated research on neuropsychiatric disorder grant in the Strategic Research Program for Brain Sciences by the Ministry of Education, Culture, Sports, Science, and Technology of Japan (to K.N.). We also thank the technical services provided by the Bio-image Core Facility of the National Core Facility Program for Biotechnology, Ministry of Science and Technology, Taiwan.

\section{Author contributions}

Y.T.L., C.C.C., C.C.H., and K.S.H. designed experiments; Y.T.L. and C.C.C. performed experiments and analyzed data; K.N. provided OXTR-Venus knock-in mice. Y.T.L., C.C. C., C.C.H., and K.S.H. wrote the paper.

\section{Additional information}

Supplementary Information accompanies this paper at doi:10.1038/s41467-017-00675-5. 
Competing interests: The authors declare no competing financial interests.

Reprints and permission information is available online at http://npg.nature.com/ reprintsandpermissions/

Publisher's note: Springer Nature remains neutral with regard to jurisdictional claims in published maps and institutional affiliations.

Open Access This article is licensed under a Creative Commons Attribution 4.0 International License, which permits use, sharing, adaptation, distribution and reproduction in any medium or format, as long as you give appropriate credit to the original author(s) and the source, provide a link to the Creative Commons license, and indicate if changes were made. The images or other third party material in this article are included in the article's Creative Commons license, unless indicated otherwise in a credit line to the material. If material is not included in the article's Creative Commons license and your intended use is not permitted by statutory regulation or exceeds the permitted use, you will need to obtain permission directly from the copyright holder. To view a copy of this license, visit http://creativecommons.org/ licenses/by/4.0/.

(c) The Author(s) 2017 